\title{
Medium- and high-spin band structure of the chiral-candidate nucleus ${ }^{134} \mathrm{Pr}$
}

\author{
J. Timár, ${ }^{1}$ K. Starosta, ${ }^{2,3}$ I. Kuti, ${ }^{1}$ D. Sohler, ${ }^{1}$ D. B. Fossan, ${ }^{2}$ T. Koike, ${ }^{2,4}$ E. S. Paul, ${ }^{5}$ A. J. Boston, ${ }^{5}$ H. J. Chantler, ${ }^{5}$ \\ M. Descovich, ${ }^{5}$ R. M. Clark, ${ }^{6}$ M. Cromaz, ${ }^{6}$ P. Fallon, ${ }^{6}$ I. Y. Lee, ${ }^{6}$ A. O. Macchiavelli, ${ }^{6}$ C. J. Chiara, ${ }^{2,7,8}$ R. Wadsworth, ${ }^{9}$ \\ A. A. Hecht, ${ }^{10}$ D. Almehed, ${ }^{11}$ and S. Frauendorf ${ }^{11}$ \\ ${ }^{1}$ Institute of Nuclear Research, Pf. 51, HU-4001 Debrecen, Hungary \\ ${ }^{2}$ Department of Physics and Astronomy, State University of New York at Stony Brook, Stony Brook, New York 11794-3800, USA \\ ${ }^{3}$ Department of Chemistry, Simon Fraser University, Burnaby, British Columbia V5A 1S6, Canada \\ ${ }^{4}$ Graduate School of Science, Tohoku University, Sendai, 980-8578, Japan \\ ${ }^{5}$ Oliver Lodge Laboratory, Department of Physics, University of Liverpool, Liverpool L69 7ZE, UK \\ ${ }^{6}$ Nuclear Science Division, Lawrence Berkeley National Laboratory, Berkeley, California 94720, USA \\ ${ }^{7}$ Department of Chemistry and Biochemistry, University of Maryland, College Park, Maryland 20742, USA \\ ${ }^{8}$ Physics Division, Argonne National Laboratory, Argonne, Illinois 60439, USA \\ ${ }^{9}$ Department of Physics, University of York, York YO10 5DD, UK \\ ${ }^{10}$ Wright Nuclear Structure Laboratory, Yale University, New Haven, Connecticut 06520, USA \\ ${ }^{11}$ Physics Department, University of Notre Dame, Notre Dame, Indiana 46556, USA
}

(Received 23 May 2011; published 5 October 2011)

\begin{abstract}
Medium- and high-spin states of ${ }^{134} \mathrm{Pr}$ were populated using the ${ }^{116} \mathrm{Cd}\left({ }^{23} \mathrm{Na}, 5 n\right)$ reaction and studied with the GAMMASPHERE spectrometer. Several new bands have been found in this nucleus, one of them being linked to the previously observed chiral-candidate twin-band structure. The ground state of ${ }^{134} \operatorname{Pr}$ could be determined through establishing a level structure that connects the two previously known long-lived isomeric states. Unambiguous spin-parity assignments for the excited states could be performed based on the known $2^{-}$spin-parity of the ground state combined with the present experimental data. Intrinsic single-particle configurations have been assigned to the newly observed bands on the basis of the measured $B(M 1) / B(E 2)$ ratios, alignments, band-crossing frequencies, bandhead spins, the observed single-particle configurations in the neighboring nuclei, and taking into account the predictions of total Routhian surface and tilted-axis cranking calculations.
\end{abstract}

DOI: 10.1103/PhysRevC.84.044302

PACS number(s): 21.10.Hw, 21.10.Re, 21.60.-n, 23.20.Lv

\section{INTRODUCTION}

Unambiguous identification of intrinsic chirality in rotating triaxial nuclei is one of the most intriguing tasks of contemporary high-spin nuclear structure studies. Intrinsic chirality is generated when the total angular momentum vector of a rotating triaxial nucleus is out of the three symmetry planes of the triaxial mean field [1,2]. According to recent model calculations the experimental manifestation of this intrinsic chirality is a structure of two, almost degenerate, $\Delta I=1$ rotational bands having equal parity and linked to each other by inter-band $\gamma$-ray transitions. Such rotational doublet-band candidates for chiral structures have been observed mostly in two regions of the nuclear chart: around ${ }^{134} \operatorname{Pr}$ (see, e.g., Refs. [3-16]) and around ${ }^{104} \mathrm{Rh}$ (see, e.g., Refs. [17-22]).

One of the key nuclei among the chiral candidates is ${ }^{134} \mathrm{Pr}$. The strongly linked $\Delta I=1$ rotational band structure, found by Petrache et al. [3] and assigned to the $\pi h_{11 / 2} v h_{11 / 2}$ configuration, was the first experimental candidate for chiral rotation [1]. Since the publication of the chiral hypothesis for ${ }^{134} \mathrm{Pr}$, this nucleus has been a subject of many experimental and theoretical studies [4,9,15,23-27] providing several different scenarios for the nature of the chiral-candidate band structure. However, the many studies published up to now on ${ }^{134} \mathrm{Pr}$ concentrated mainly on the known chiral-candidate band structure in this nucleus, and thus, in many experimental aspects, the level scheme of this nucleus cannot be considered well known.

High-spin rotational bands in this nucleus were reported by Beausang et al. [28], Petrache et al. [3], and Hauschild et al. [29]. Besides the chiral-candidate band structure, three additional rotational bands were reported. These include a negative-parity four-quasiparticle band, assigned the $\pi[413] 5 / 2\left(h_{11 / 2}\right)^{2} v h_{11 / 2}$ configuration, in addition to two doubly decoupled bands, assigned to $\pi h_{11 / 2} v[530] 1 / 2$ and $\pi h_{11 / 2} \nu[660] 1 / 2$ configurations, respectively. The latter could not be linked to the rest of the level scheme and has a tentative parity assignment. The lifetimes of levels in the doubly decoupled bands were later measured by Rao et al. [30], while the lifetimes of the chiral-candidate band structure were reported by Tonev et al. [23].

No negative-parity two-quasiparticle bands have been reported in this nucleus, although they are expected. Also, the problem of deciding which of the two long-lived isomeric states is the ground state and which is the excited state has not been solved. Moreover, even for the known states and bands, the reported spins and parities are assigned tentatively. The aim of the present paper is to establish a more detailed level scheme of ${ }^{134} \mathrm{Pr}$ to enrich our knowledge of the active valence particles and the shape stability of this nucleus. This information is relevant also to studies addressing the chiral-candidate band structure. 


\section{EXPERIMENTAL METHODS AND RESULTS}

Excited states in ${ }^{134} \mathrm{Pr}$ were populated following the ${ }^{116} \mathrm{Cd}\left({ }^{23} \mathrm{Na}, 5 n\right)$ reaction at a beam energy of $115 \mathrm{MeV}$. The experiment was performed at the Lawrence Berkeley National Laboratory, using a ${ }^{23} \mathrm{Na}$ beam supplied by the 88-inch cyclotron. The experimental setup consisted of the GAMMASPHERE spectrometer of 99 HPGe detectors in 16 rings covering a large fraction of the full solid angle [31,32] (with ring 1 at $\theta=17.3^{\circ}$ being empty). In order to obtain $\gamma$-ray sum-energy and multiplicity information, the Hevimet collimators ordinarily placed in front of the BGO Comptonsuppression shields were removed for this experiment as discussed in Ref. [33]. The sum-energy and multiplicity data were not used in the present analysis; however, they were important in studying other nuclei from this experiment [34].

The experiment consisted of two runs: Run 1 uses a $1.00 \mathrm{mg} / \mathrm{cm}^{2}$ thick ${ }^{116} \mathrm{Cd}$ target backed with $15 \mathrm{mg} / \mathrm{cm}^{2}$ ${ }^{208} \mathrm{~Pb}$ aimed at performing lifetime measurements using the Doppler shift attenuation method (DSAM) [35], while Run 2 uses a self-supporting $1.22 \mathrm{mg} / \mathrm{cm}^{2}$ thick ${ }^{116} \mathrm{Cd}$ target aimed to identify the high-spin states in ${ }^{134} \mathrm{Pr}$. The current paper reports results from both runs; however, the DSAM analysis will be addressed in a separate paper.

For the off-line analysis, coincident $\gamma$ rays were sorted using the BLUE and RADWARE software packages following the procedures defined in Refs. [36] and [37], respectively. The details of the data analysis with the BLUE package, especially the handling of background subtraction for the DSAM analysis, are addressed in Ref. [38]. The $\gamma$-ray energies observed in Run 2 were corrected for Doppler shifts. The average velocity of the recoiling nuclei was measured to be $\beta \sim 1.380(5) \%$, in agreement with the value calculated based on reaction kinematics. For Run 2 a $\gamma$-ray energy resolution of $\Delta E_{\gamma} \sim 3.8 \mathrm{keV}$ full width at half maximum (FWHM) at $E_{\gamma} \sim 660 \mathrm{keV}$ was achieved; the resolution was measured using the $19 / 2^{-} \rightarrow 15 / 2^{-}$transition in ${ }^{133} \mathrm{Pr}$.

An angular-correlation analysis, based on the directional correlation of oriented states (DCO) method [39] was performed for $\gamma$-ray multipolarity assignments. This analysis was carried out based on the spectra extracted from the BLUE database for the backed target Run 1 with the background subtracted according to the prescription of Ref. [38]. In the geometry used, $R_{\mathrm{DCO}}$ values of 1 and $\sim 0.6$ are expected for the stretched quadrupole $(\Delta I=2)$ and pure stretched dipole $(\Delta I=1)$ transitions, respectively, when gating on a quadrupole transition. Using a stretched dipole gate with minimal quadrupole mixing, the expected values are $\sim 1.7$ and $\sim 1$, respectively. The expected $R_{\mathrm{DCO}}$ values for $\Delta I=0$ dipole transitions are similar to the ones for the stretched quadrupole transitions.

In order to analyze the $\gamma$-ray coincidence relations and to build the level scheme of ${ }^{134} \mathrm{Pr}$ the collected events were sorted into RADWARE cubes separately for the thin-target data and the thick-target data. They contained approximately $7 \times 10^{9}$ and $9 \times 10^{9}$ triple coincidences, respectively. The good statistics of the experiment enabled us to assign a large number of new $\gamma$ rays to ${ }^{134} \mathrm{Pr}$ and determine their multipolarities. Altogether approximately $360 \gamma$ transitions have been assigned to this nucleus, more than half of them being identified for the first time in the present experiment. The properties of the observed ${ }^{134} \operatorname{Pr} \gamma$ rays are given in Table I. In the table, we accepted E2 multipolarity for all the $\gamma$ rays with $R_{\mathrm{DCO}}$ values corresponding to quadrupole transitions. If the transition is a stretched dipole on the basis of its $R_{\mathrm{DCO}}$ value, we took into account other possible arguments in order to determine its electric and magnetic character. For example, for an in-band stretched dipole transition, we accepted $M 1$ multipolarity. Example double-gated, background-subtracted coincidence spectra are plotted in Fig. 1.

\section{LEVEL SCHEME}

From the observed $\gamma \gamma \gamma$-coincidence relations we have built a more complete level scheme of ${ }^{134} \mathrm{Pr}$. The observed data also enabled us to determine which of the two long-lived isomeric states is the ground state of ${ }^{134} \mathrm{Pr}$ and to obtain unambiguous spin and parity assignments for the excited states of this nucleus, which were only tentatively known before the present experiment. The derived level scheme contains 13 bands, among which 9 are newly assigned to ${ }^{134} \mathrm{Pr}$. For the sake of clarity the level scheme is plotted in Figs. 2, 3, and 4, respectively.

\section{A. Low- and medium-spin negative-parity bands}

Two long-lived isomeric states are known in ${ }^{134} \mathrm{Pr}$. One has a 17-min lifetime and its spin-parity is unambiguously determined as $2^{-}$from the $\beta$ decay of ${ }^{134} \mathrm{Nd}$ [40]. The other has an 11-min lifetime and was observed in heavyion-induced reactions; however, its spin-parity could not be unambiguously determined. Possible $5^{-}, 7^{-}$, and $6^{-}$values have been suggested for its spin-parity in Refs. [41-43]. The energy difference between the two isomers was unknown up to the present experiment. Petrache et al. [3] observed the $M 1$ cascade of band 12 and other $\gamma$ transitions in coincidence with that cascade. They realized that this cascade feeds the $2^{-}$isomeric state and that it is connected to band 5; however, they could not establish the corresponding level structure due to lack of statistics.

Five new negative-parity bands at low to medium spins (bands 9, 10, 11, 12, and 13 in Fig. 2) have been derived from the present experiment. Band 12 decays by the 332and the $614-\mathrm{keV}$ transitions to the state which is identified from the previous heavy-ion reaction studies as the 11-min isomeric state. However, the bandhead of band 12 lies $68 \mathrm{keV}$ lower than this state, and this is the lowest-lying level in the observed level scheme. Therefore, it is straightforward to identify this bandhead as the 17-min isomeric state. Thus, these linking transitions fix the relative position of the two isomeric states, with the 17-min state being the ground state and the 11-min state having an excitation energy of $68 \mathrm{keV}$. The five bands shown in Fig. 2 are linked together through many $\gamma$ transitions, and there are also several other paths that link the two isomeric states together, thus confirming the relative positions stated above. 
TABLE I. Energies, relative intensities, DCO ratios, and multipolarities of $\gamma$ rays assigned to ${ }^{134} \mathrm{Pr}$ in the present work, as well as the band assignment for the initial state, the spin-parities of the initial and final states, and the excitation energy of the initial state for the decay. The experimental errors of the relative intensities for the strong and/or well-resolved transitions are on the order of 5\% while for weak or compound lines the errors can rise up to $50 \%$. DCO ratios were determined using quadrupole gates if not indicated otherwise. $l s$ denotes the small level structure between bands 10 and 11 . D denotes dipole transition.

\begin{tabular}{|c|c|c|c|c|c|c|c|c|}
\hline$E_{\gamma}(\mathrm{keV})$ & $I_{\gamma}$ & $R_{\mathrm{DCO}}$ & Mult. & Band $_{i}$ & $I_{i}^{\pi}$ & $\rightarrow$ & $I_{f}^{\pi}$ & $E_{i}(\mathrm{keV})$ \\
\hline $39.3(3)$ & 21.5 & & $M 1^{\mathrm{a}}$ & 1 & $8^{+}$ & $\rightarrow$ & $7^{+}$ & 414 \\
\hline $46.5(4)$ & 0.1 & & & 11 & $7^{-}$ & $\rightarrow$ & $6^{-}$ & 751 \\
\hline $82.4(4)$ & 0.4 & & $E 1^{\mathrm{b}}$ & 1 & $7^{+}$ & $\rightarrow$ & $7^{-}$ & 374 \\
\hline $90.7(3)$ & 0.7 & & & 9 & $7^{-}$ & $\rightarrow$ & $7^{-}$ & 383 \\
\hline $94.6(3)$ & 39.2 & $0.74(5)$ & $M 1^{\mathrm{a}}$ & 1 & $9^{+}$ & $\rightarrow$ & $8^{+}$ & 508 \\
\hline $103.1(2)$ & 0.2 & & & 9 & $8^{-}$ & $\rightarrow$ & $8^{-}$ & 653 \\
\hline $109.3(3)$ & 0.2 & & & 13 & $8^{-}$ & $\rightarrow$ & $\left(7^{-}\right)$ & 906 \\
\hline $115.7(2)$ & 5.8 & $0.57(5)$ & $M 1$ & 12 & $3^{-}$ & $\rightarrow$ & $2^{-}$ & 116 \\
\hline $117.9(2)$ & 3.8 & $0.58(5)$ & $M 1$ & 12 & $6^{-}$ & $\rightarrow$ & $5^{-}$ & 517 \\
\hline $120.7(3)$ & 0.2 & & & 11 & $6^{-}$ & $\rightarrow$ & $\left(6^{-}\right)$ & 705 \\
\hline 121.9(3) & 0.3 & & $E 1^{\mathrm{b}}$ & 1 & $8^{+}$ & $\rightarrow$ & $7^{-}$ & 414 \\
\hline $130.6(2)$ & 4.4 & & & 11 & $8^{-}$ & $\rightarrow$ & $7^{-}$ & 882 \\
\hline $131.3(2)$ & 5.0 & $0.51(4)$ & $M 1$ & 12 & $4^{-}$ & $\rightarrow$ & $3^{-}$ & 247 \\
\hline $133.9(2)$ & 0.6 & $0.43(13)$ & $M 1$ & 3 & $10^{+}$ & $\rightarrow$ & $9^{+}$ & 1306 \\
\hline $146.3(2)$ & 1.6 & $0.46(6)$ & D & 5 & $14^{-}$ & $\rightarrow$ & $13^{-}$ & 2748 \\
\hline $152.5(2)$ & 5.2 & $0.56(5)$ & $M 1$ & 12 & $5^{-}$ & $\rightarrow$ & $4^{-}$ & 400 \\
\hline $164.5(2)$ & 3.6 & $0.64(6)$ & $M 1$ & 12 & $7^{-}$ & $\rightarrow$ & $6^{-}$ & 682 \\
\hline $166.4(2)$ & 1.1 & & & 2 & $11^{+}$ & $\rightarrow$ & $10^{+}$ & 1304 \\
\hline $166.9(3)$ & 0.1 & & & 11 & $7^{-}$ & $\rightarrow$ & $\left(6^{-}\right)$ & 751 \\
\hline $167.5(4)$ & 0.2 & & & 2 & $9^{+}$ & $\rightarrow$ & $8^{+}$ & 933 \\
\hline $171.2(2)$ & 71.9 & $0.55(2)$ & $M 1$ & 1 & $10^{+}$ & $\rightarrow$ & $9^{+}$ & 679 \\
\hline 171.3(3) & 0.2 & & & 2 & $10^{+}$ & $\rightarrow$ & $11^{+}$ & 1138 \\
\hline $179.2(2)$ & 0.8 & $1.59(24)^{c}$ & D & 11 & $9^{-}$ & $\rightarrow$ & $8^{-}$ & 1086 \\
\hline $184.7(3)$ & 0.7 & & & $l s$ & $\left(6^{-}\right)$ & $\rightarrow$ & $5^{-}$ & 584 \\
\hline $187.3(3)$ & 0.1 & & & 13 & $10^{-}$ & $\rightarrow$ & $\left(9^{-}\right)$ & 1468 \\
\hline 199.8(3) & 0.5 & $0.93(27)^{c}$ & $M 1$ & 6 & $14^{-}$ & $\rightarrow$ & $13^{-}$ & 2910 \\
\hline $200.6(2)$ & 12.7 & $0.57(9)$ & $M 1$ & 5 & $14^{-}$ & $\rightarrow$ & $13^{-}$ & 2748 \\
\hline 204.0(3) & 2.7 & $0.90(6)^{c}$ & $M 1$ & 11 & $9^{-}$ & $\rightarrow$ & $8^{-}$ & 1086 \\
\hline $204.6(2)$ & 17.3 & $0.63(7)$ & $M 1$ & 5 & $15^{-}$ & $\rightarrow$ & $14^{-}$ & 2953 \\
\hline $204.8(2)$ & 0.8 & & & 2 & $10^{+}$ & $\rightarrow$ & $9^{+}$ & 1138 \\
\hline $210.7(2)$ & 1.7 & $0.46(5)$ & $M 1$ & 3 & $11^{+}$ & $\rightarrow$ & $10^{+}$ & 1516 \\
\hline $212.8(3)$ & 0.2 & & & $l s$ & $\left(7^{-}\right)$ & $\rightarrow$ & $\left(6^{-}\right)$ & 797 \\
\hline $215.8(2)$ & 0.7 & & & 10 & $9^{-}$ & $\rightarrow$ & $8^{-}$ & 869 \\
\hline $216.3(2)$ & 1.3 & & & 5 & $12^{-}$ & $\rightarrow$ & $11^{-}$ & 2317 \\
\hline $224.3(2)$ & 14.6 & & & 10 & $7^{-}$ & $\rightarrow$ & $6^{-}$ & 292 \\
\hline $224.7(2)$ & 1.0 & $0.53(10)$ & D & 13 & $8^{-}$ & $\rightarrow$ & $7^{-}$ & 906 \\
\hline $225.0(6)$ & 0.6 & & & 12 & $6^{-}$ & $\rightarrow$ & $7^{-}$ & 517 \\
\hline $227.5(2)$ & 1.5 & $1.06(19)^{c}$ & $M 1$ & 6 & $15^{-}$ & $\rightarrow$ & $14^{-}$ & 3137 \\
\hline $230.4(2)$ & 4.2 & $0.32(13)$ & $M 1$ & 5 & $13^{-}$ & $\rightarrow$ & $12^{-}$ & 2548 \\
\hline $233.9(3)$ & 1.3 & $0.79(8)^{c}$ & $\mathrm{D}$ & 11 & $7^{-}$ & $\rightarrow$ & $6^{-}$ & 751 \\
\hline 239.6(3) & 0.3 & & & 10 & $11^{-}$ & $\rightarrow$ & $10^{-}$ & 1573 \\
\hline $240.0(2)$ & 19.2 & & & 5 & $16^{-}$ & $\rightarrow$ & $15^{-}$ & 3193 \\
\hline $243.3(2)$ & 0.4 & $0.43(19)$ & D & 3 & $11^{+}$ & $\rightarrow$ & $12^{+}$ & 1516 \\
\hline $245.0(2)$ & 1.6 & $0.51(6)$ & D & 4 & $11^{+}$ & $\rightarrow$ & $10^{+}$ & 1551 \\
\hline 246.6(4) & 0.2 & & & 10 & $13^{-}$ & $\rightarrow$ & $12^{-}$ & 2380 \\
\hline $250.2(2)$ & 0.8 & & & 3 & $13^{+}$ & $\rightarrow$ & $12^{+}$ & 2034 \\
\hline 251.1(2) & 2.9 & $0.37(8)$ & D & 13 & $9^{-}$ & $\rightarrow$ & $8^{-}$ & 1133 \\
\hline 253.4(4) & 0.9 & & & 11 & $10^{-}$ & $\rightarrow$ & $9^{-}$ & 1386 \\
\hline $253.6(4)$ & 0.2 & & & 2 & $9^{+}$ & $\rightarrow$ & $10^{+}$ & 933 \\
\hline
\end{tabular}

TABLE I. (Continued.)

\begin{tabular}{|c|c|c|c|c|c|c|c|c|}
\hline$E_{\gamma}(\mathrm{keV})$ & $I_{\gamma}$ & $R_{\mathrm{DCO}}$ & Mult. & Band $_{i}$ & $I_{i}^{\pi}$ & $\rightarrow$ & $I_{f}^{\pi}$ & $E_{i}(\mathrm{keV})$ \\
\hline 257.1(3) & 0.2 & & & 2 & $8^{+}$ & $\rightarrow$ & $9^{+}$ & 765 \\
\hline $257.8(2)$ & 6.9 & $0.23(5)$ & $M 1$ & 10 & $8^{-}$ & $\rightarrow$ & $7^{-}$ & 550 \\
\hline $258.2(3)$ & 0.2 & & & 7 & $16^{-}$ & $\rightarrow$ & $15^{-}$ & 3545 \\
\hline 267.2(2) & 0.6 & & & 3 & $12^{+}$ & $\rightarrow$ & $11^{+}$ & 1784 \\
\hline $270.0(2)$ & 2.1 & & & 9 & $8^{-}$ & $\rightarrow$ & $7^{-}$ & 653 \\
\hline 270.3(3) & 0.4 & & & 12 & $6^{-}$ & $\rightarrow$ & $4^{-}$ & 517 \\
\hline $277.6(2)$ & 0.7 & $0.42(12)$ & D & 4 & $11^{+}$ & $\rightarrow$ & $12^{+}$ & 1551 \\
\hline $280.0(2)$ & 4.6 & $0.48(12)$ & $M 1$ & 6 & $16^{-}$ & $\rightarrow$ & $15^{-}$ & 3417 \\
\hline 283.3(2) & 6.9 & $1.33(36)^{\mathrm{c}}$ & $M 1$ & 2 & $12^{+}$ & $\rightarrow$ & $11^{+}$ & 1587 \\
\hline 283.9(4) & 0.2 & & & 12 & $5^{-}$ & $\rightarrow$ & $3^{-}$ & 400 \\
\hline $287.6(2)$ & 62.7 & & & 1 & $11^{+}$ & $\rightarrow$ & $10^{+}$ & 967 \\
\hline 289.9(2) & 20.0 & $0.65(8)$ & $M 1$ & 5 & $17^{-}$ & $\rightarrow$ & $16^{-}$ & 3483 \\
\hline $290.8(4)$ & 1.3 & & & 5 & $14^{-}$ & $\rightarrow$ & $13^{-}$ & 2748 \\
\hline 292.3(2) & 1.1 & & & 7 & $16^{-}$ & $\rightarrow$ & $15^{-}$ & 3545 \\
\hline 293.8(2) & 1.9 & $1.10(18)^{\mathrm{c}}$ & D & 6 & $15^{-}$ & $\rightarrow$ & $14^{-}$ & 3137 \\
\hline $300.8(2)$ & 2.3 & $0.30(4)$ & $M 1$ & 11 & $10^{-}$ & $\rightarrow$ & $9^{-}$ & 1386 \\
\hline 305.3(3) & 1.0 & & & 11 & $6^{-}$ & $\rightarrow$ & $5^{-}$ & 705 \\
\hline $306.5(3)$ & 40.4 & & & 1 & $12^{+}$ & $\rightarrow$ & $11^{+}$ & 1273 \\
\hline $306.6(2)$ & 100.0 & 0 & D & 1 & $7^{+}$ & $\rightarrow$ & $6^{-}$ & \\
\hline $307.6(3)$ & 0.3 & & & 9 & $10^{-}$ & $\rightarrow$ & $9^{-}$ & 1333 \\
\hline $315.0(2)$ & 1.5 & $0.34(14)$ & D & 9 & $7^{-}$ & $\rightarrow$ & $6^{-}$ & 383 \\
\hline $316.5(2)$ & 1.4 & & & 7 & $17^{-}$ & $\rightarrow$ & $16^{-}$ & 3861 \\
\hline $318.8(2)$ & 5.4 & & & 10 & $9^{-}$ & $\rightarrow$ & $8^{-}$ & 869 \\
\hline $318.9(2)$ & 5.0 & & & 6 & $17^{-}$ & $\rightarrow$ & $16^{-}$ & 3736 \\
\hline $323.9(2)$ & 14.6 & $0.49(7)$ & $M 1$ & 2 & $13^{+}$ & $\rightarrow$ & $12^{+}$ & 1911 \\
\hline $324.4(3)$ & 0.2 & & & 10 & $15^{-}$ & $\rightarrow$ & $14^{-}$ & 3286 \\
\hline $331.8(3)$ & 1.3 & $0.48(10)$ & D & 12 & $5^{-}$ & $\rightarrow$ & $6^{-}$ & 400 \\
\hline $332.1(5)$ & 0.4 & & & 11 & $8^{-}$ & $\rightarrow$ & $8^{-}$ & 32 \\
\hline $332.5(2)$ & 1.6 & $0.53(11)$ & $M 1$ & 11 & $11^{-}$ & $\rightarrow$ & $10^{-}$ & 1719 \\
\hline $333.8(4)$ & 0.4 & & & 10 & $13^{-}$ & $\rightarrow$ & $12^{-}$ & 2380 \\
\hline $334.4(3)$ & 0.4 & & & 11 & $9^{-}$ & $\rightarrow$ & $7^{-}$ & 1086 \\
\hline 33 & 0.4 & $1.11(38)^{\mathrm{c}}$ & $M 1$ & 13 & $10^{-}$ & $\rightarrow$ & $9^{-}$ & 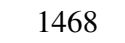 \\
\hline $337.1(2)$ & 13.1 & $0.46(10)$ & $M 1$ & 2 & $14^{+}$ & $\rightarrow$ & $13^{+}$ & 2248 \\
\hline $337.6(4)$ & 1.0 & & & 11 & $7^{-}$ & $\rightarrow$ & $8^{+}$ & 751 \\
\hline $339.2(2)$ & 2.3 & 0.64 & D & 3 & $10^{+}$ & $\rightarrow$ & $11^{+}$ & 1306 \\
\hline 33 & 1.2 & 0.2 & $M 1$ & 10 & $11^{-}$ & $\rightarrow$ & 10 & 573 \\
\hline $339.8(2)$ & 1.2 & $0.49(22)$ & $M 1$ & 7 & $18^{-}$ & $\rightarrow$ & $17^{-}$ & 4201 \\
\hline $343.0(2)$ & 15.4 & $0.61(10)$ & $M 1$ & 5 & $18^{-}$ & $\rightarrow$ & $17^{-}$ & 3826 \\
\hline 35810 & 1.2 & & & 3 & $14^{+}$ & $\rightarrow$ & $13^{+}$ & 2392 \\
\hline $359.8(2)$ & 4.0 & $0.95(17)^{\mathrm{c}}$ & $M 1$ & 6 & $18^{-}$ & $\rightarrow$ & $17^{-}$ & 4096 \\
\hline 364.1(2) & 1.1 & & & 10 & $10^{-}$ & $\rightarrow$ & $9^{-}$ & 1233 \\
\hline $364.5(3)$ & 0.5 & & & 11 & $8^{-}$ & $\rightarrow$ & $6^{-}$ & \\
\hline 36 & 0.2 & & & 4 & $9^{+}$ & $\rightarrow$ & 8 & 11 \\
\hline $368.3(2)$ & 1.2 & $0.42(11)$ & D & 5 & $14^{-}$ & $\rightarrow$ & $13^{-}$ & 2748 \\
\hline $369.2(2)$ & 6.1 & & & 2 & $15^{+}$ & $\rightarrow$ & $14^{+}$ & 2617 \\
\hline 37 & 0.7 & & & & $9^{-}$ & $\rightarrow$ & $8^{-}$ & 1026 \\
\hline $9(4)$ & 0.5 & & & 11 & $7^{-}$ & $\rightarrow$ & $7^{+}$ & \\
\hline $377.8(3)$ & 0.3 & & & 4 & $13^{+}$ & $\rightarrow$ & $12^{+}$ & 2161 \\
\hline $.5(3)$ & 0.4 & $0.31(17)$ & $M 1$ & 1 & $12^{-}$ & $\rightarrow$ & $11^{-}$ & 2158 \\
\hline 38170 & 1.0 & & & & $9^{-}$ & $\rightarrow$ & $7^{-}$ & \\
\hline 382.1(3) & 0.3 & & & 15 & $10^{-}$ & $\rightarrow$ & $9^{-}$ & 1468 \\
\hline $382.5(3)$ & 1.6 & 0.40 & $M 1$ & 11 & $12^{-}$ & $\rightarrow$ & $11^{-}$ & 2101 \\
\hline 386.1(2) & 10.7 & & $M 1$ & 5 & $19^{-}$ & 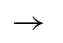 & $18-$ & 4212 \\
\hline & 0.8 & & $M 1$ & 1 & $14^{-}$ & $\rightarrow$ & $13^{-}$ & 2844 \\
\hline 389.4(4) & 0.8 & $0.98(15)$ & $E 2$ & 13 & $8^{-}$ & $\rightarrow$ & $6^{-}$ & 906 \\
\hline $389.8(3)$ & 1.2 & & & 5 & $13^{-}$ & $\rightarrow$ & $12^{-}$ & 2548 \\
\hline & 0.1 & & & 2 & $8^{+}$ & $\rightarrow$ & $7^{+}$ & 765 \\
\hline 393.0(3) & 1.2 & & & 13 & $11^{-}$ & $\rightarrow$ & $10^{-}$ & 1779 \\
\hline
\end{tabular}


TABLE I. (Continued.)

\begin{tabular}{|c|c|c|c|c|c|c|c|c|}
\hline$E_{\gamma}(\mathrm{keV})$ & $I_{\gamma}$ & $R_{\mathrm{DCO}}$ & Mult. & Band $_{i}$ & $I_{i}^{\pi}$ & $\rightarrow$ & $I_{f}^{\pi}$ & $E_{i}(\mathrm{keV})$ \\
\hline $395.6(2)$ & 13.8 & & & 1 & $14^{+}$ & $\rightarrow$ & $13^{+}$ & 2085 \\
\hline $396.9(2)$ & 1.3 & $0.39(23)$ & D & 8 & $19^{-}$ & $\rightarrow$ & $18^{-}$ & 4598 \\
\hline $400.0(2)$ & 3.5 & & & 2 & $16^{+}$ & $\rightarrow$ & $15^{+}$ & 3017 \\
\hline $403.8(2)$ & 2.7 & $2.18(17)^{\mathrm{c}}$ & $E 2$ & 11 & $9^{-}$ & $\rightarrow$ & $7^{-}$ & 1086 \\
\hline 405.1(5) & 0.1 & & & 5 & $15^{-}$ & $\rightarrow$ & $13^{-}$ & 2953 \\
\hline $410.8(2)$ & 2.7 & $0.79(22)^{c}$ & $M 1$ & 6 & $19^{-}$ & $\rightarrow$ & $18^{-}$ & 4507 \\
\hline $412.9(3)$ & 0.6 & & & 11 & $6^{-}$ & $\rightarrow$ & $7^{-}$ & 705 \\
\hline $416.6(2)$ & 25.8 & & & 1 & $13^{+}$ & $\rightarrow$ & $12^{+}$ & 1690 \\
\hline $421.3(2)$ & 0.8 & $1.65(67)$ & $E 2$ & 4 & $11^{+}$ & $\rightarrow$ & $9^{+}$ & 1551 \\
\hline $421.6(2)$ & 1.9 & & & 6 & $20^{-}$ & $\rightarrow$ & $19^{-}$ & 4928 \\
\hline $425.6(3)$ & 0.8 & & & 7 & $20^{-}$ & $\rightarrow$ & $19^{-}$ & 5059 \\
\hline $427.5(4)$ & 0.1 & & & 6 & $15^{-}$ & $\rightarrow$ & $13^{-}$ & 3137 \\
\hline $430.8(4)$ & 0.5 & & & 5 & $14^{-}$ & $\rightarrow$ & $12^{-}$ & 2748 \\
\hline $432.7(2)$ & 1.1 & & & 7 & $19^{-}$ & $\rightarrow$ & $18^{-}$ & 4634 \\
\hline $436.5(2)$ & 3.1 & & & 2 & $16^{+}$ & $\rightarrow$ & $15^{+}$ & 3017 \\
\hline $438.4(2)$ & 6.5 & $0.50(22)$ & $M 1$ & 5 & $20^{-}$ & $\rightarrow$ & $19^{-}$ & 4650 \\
\hline $438.9(2)$ & 1.4 & & & 3 & $15^{+}$ & $\rightarrow$ & $14^{+}$ & 2831 \\
\hline $440.7(3)$ & 1.3 & & & 1 & $21^{+}$ & $\rightarrow$ & $20^{+}$ & 5609 \\
\hline $443.8(2)$ & 1.1 & & & 1 & $16^{+}$ & $\rightarrow$ & $15^{+}$ & 3061 \\
\hline 444.4(4) & 0.7 & & & 5 & $16^{-}$ & $\rightarrow$ & $14^{-}$ & 3193 \\
\hline 444.4(4) & 0.2 & & & 13 & $13^{-}$ & $\rightarrow$ & $12^{-}$ & 2602 \\
\hline $445.1(2)$ & 1.3 & & & 3 & $16^{+}$ & $\rightarrow$ & $15^{+}$ & 3276 \\
\hline $445.6(3)$ & 0.5 & & & 3 & $17^{+}$ & $\rightarrow$ & $16^{+}$ & 3722 \\
\hline $446.2(3)$ & 0.9 & & & 5 & $13^{-}$ & $\rightarrow$ & $12^{-}$ & 2548 \\
\hline 446.4(4) & 0.2 & & & 5 & $13^{-}$ & $\rightarrow$ & $11^{-}$ & 2548 \\
\hline $450.4(2)$ & 0.8 & $0.59(28)$ & $\mathrm{D}$ & 4 & $9^{+}$ & $\rightarrow$ & $10^{+}$ & 1130 \\
\hline $451.1(2)$ & 0.8 & $1.81(25)^{\mathrm{c}}$ & $E 2$ & 13 & $9^{-}$ & $\rightarrow$ & $7^{-}$ & 1133 \\
\hline $458.7(2)$ & 3.8 & & & 1 & $11^{+}$ & $\rightarrow$ & $9^{+}$ & 967 \\
\hline $459.5(3)$ & 1.5 & & & 11 & $7^{-}$ & $\rightarrow$ & $7^{-}$ & 751 \\
\hline $463.6(4)$ & 0.1 & & & 11 & $14^{-}$ & $\rightarrow$ & $13^{-}$ & 2844 \\
\hline $464.7(3)$ & 0.7 & & & 9 & $10^{-}$ & $\rightarrow$ & $9^{-}$ & 1333 \\
\hline $467.2(3)$ & 2.1 & & & 2 & $18^{+}$ & $\rightarrow$ & $17^{+}$ & 3956 \\
\hline $472.0(2)$ & 3.6 & $0.68(19)^{c}$ & $M 1$ & 2 & $17^{+}$ & $\rightarrow$ & $16^{+}$ & 3489 \\
\hline $473.4(3)$ & 1.1 & & & 1 & $22^{+}$ & $\rightarrow$ & $21^{+}$ & 6082 \\
\hline $473.5(4)$ & 0.5 & & & 10 & $12^{-}$ & $\rightarrow$ & $11^{-}$ & 2046 \\
\hline 474.4(3) & 0.6 & & & 7 & $21^{-}$ & $\rightarrow$ & $20^{-}$ & 5533 \\
\hline $475.9(3)$ & 0.5 & & & 2 & $20^{+}$ & $\rightarrow$ & $19^{+}$ & 5022 \\
\hline $476.2(4)$ & 0.6 & & & 1 & $23^{+}$ & $\rightarrow$ & $22^{+}$ & 6558 \\
\hline $476.4(2)$ & 3.8 & & & 5 & $21^{-}$ & $\rightarrow$ & $20^{-}$ & 5126 \\
\hline 477.3(4) & 0.3 & & & 6 & $22^{-}$ & $\rightarrow$ & $21^{-}$ & 5912 \\
\hline 479.5(4) & 0.2 & & & 3 & $12^{+}$ & $\rightarrow$ & $11^{+}$ & 1784 \\
\hline $479.9(5)$ & 0.3 & & & 3 & $19^{+}$ & $\rightarrow$ & $18^{+}$ & 4713 \\
\hline 480.3(2) & 1.7 & & & 1 & $16^{+}$ & $\rightarrow$ & $15^{+}$ & 3061 \\
\hline $482.1(2)$ & 4.0 & & & 10 & $8^{-}$ & $\rightarrow$ & $6^{-}$ & 550 \\
\hline $483.5(3)$ & 0.2 & & & $l s$ & $\left(9^{-}\right)$ & $\rightarrow$ & $\left(7^{-}\right)$ & 1281 \\
\hline 492.8(2) & 0.6 & & & 3 & $9^{+}$ & $\rightarrow$ & $10^{+}$ & 1172 \\
\hline $495.7(2)$ & 7.7 & $0.39(6)$ & $M 1$ & 1 & $15^{+}$ & $\rightarrow$ & $14^{+}$ & 2581 \\
\hline 499.5(3) & 1.1 & & & 1 & $20^{+}$ & $\rightarrow$ & $19^{+}$ & 5168 \\
\hline $500.6(4)$ & 0.2 & & & 13 & $13^{-}$ & $\rightarrow$ & $12^{-}$ & 2602 \\
\hline $504.7(2)$ & 1.5 & $2.12(45)^{\mathrm{c}}$ & $E 2$ & 11 & $10^{-}$ & $\rightarrow$ & $8^{-}$ & 1386 \\
\hline $505.7(3)$ & 1.2 & & & 6 & $21^{-}$ & $\rightarrow$ & $20^{-}$ & 5434 \\
\hline $507.5(3)$ & 0.3 & & & 6 & $16^{-}$ & $\rightarrow$ & $14^{-}$ & 3417 \\
\hline $511.2(3)$ & 0.7 & & & 3 & $18^{+}$ & $\rightarrow$ & $17^{+}$ & 4233 \\
\hline $517.8(2)$ & 2.4 & & & 5 & $22^{-}$ & $\rightarrow$ & $21^{-}$ & 5644 \\
\hline $518.8(2)$ & 0.8 & & & 2 & $9^{+}$ & $\rightarrow$ & $8^{+}$ & 933 \\
\hline $521.8(2)$ & 1.2 & & & 1 & $18^{+}$ & $\rightarrow$ & $17^{+}$ & 4129 \\
\hline $523.9(4)$ & 0.5 & & & 1 & $25^{+}$ & $\rightarrow$ & $24^{+}$ & 7610 \\
\hline $528.0(4)$ & 0.6 & & & 1 & $24^{+}$ & $\rightarrow$ & $23^{+}$ & 7086 \\
\hline
\end{tabular}

TABLE I. (Continued.)

\begin{tabular}{|c|c|c|c|c|c|c|c|c|}
\hline$E_{\gamma}(\mathrm{keV})$ & $I_{\gamma}$ & $R_{\mathrm{DCO}}$ & Mult. & Band $_{i}$ & $I_{i}^{\pi}$ & $\rightarrow$ & $I_{f}^{\pi}$ & $E_{i}(\mathrm{keV})$ \\
\hline $529.8(3)$ & 1.1 & & & 5 & $17^{-}$ & $\rightarrow$ & $15^{-}$ & 3483 \\
\hline 532.1(2) & 2.1 & & & 2 & $15^{+}$ & $\rightarrow$ & $14^{+}$ & 2617 \\
\hline $539.1(3)$ & 1.2 & & & 1 & $19^{+}$ & $\rightarrow$ & $18^{+}$ & 4669 \\
\hline $540.7(2)$ & 0.3 & & & 3 & $10^{+}$ & $\rightarrow$ & $8^{+}$ & 1306 \\
\hline $546.5(2)$ & 3.1 & $0.70(22)$ & $M 1$ & 1 & $17^{+}$ & $\rightarrow$ & $16^{+}$ & 3608 \\
\hline $549.9(3)$ & 1.3 & & & 5 & $23^{-}$ & $\rightarrow$ & $22^{-}$ & 6194 \\
\hline $558.5(2)$ & 1.4 & & & 2 & $14^{+}$ & $\rightarrow$ & $13^{+}$ & 2248 \\
\hline $560.4(5)$ & 0.2 & & & 9 & $12^{-}$ & $\rightarrow$ & $11^{-}$ & 2133 \\
\hline 561.4(3) & 0.9 & & & 13 & $10^{-}$ & $\rightarrow$ & $8^{-}$ & 1468 \\
\hline $562.5(4)$ & 0.4 & & & , & $23^{-}$ & $\rightarrow$ & $22^{-}$ & 6474 \\
\hline $572.6(3)$ & 0.4 & & & 5 & $15^{-}$ & $\rightarrow$ & $13^{-}$ & 2953 \\
\hline $574.1(3)$ & 0.2 & & & 6 & $16^{-}$ & $\rightarrow$ & $14^{-}$ & 3417 \\
\hline $575.3(4)$ & 0.5 & & & 5 & $16^{-}$ & $\rightarrow$ & $15^{+}$ & 3193 \\
\hline $576.6(2)$ & 4.4 & $1.05(17)$ & $E 2$ & 10 & $9^{-}$ & $\rightarrow$ & $7^{-}$ & 869 \\
\hline $581.9(3)$ & 0.2 & & & 10 & $14^{-}$ & $\rightarrow$ & $13^{-}$ & 2962 \\
\hline 583.4(3) & 0.6 & & & 3 & $11^{+}$ & $\rightarrow$ & $9^{+}$ & 1516 \\
\hline $584.9(3)$ & 1.2 & & & 9 & $8^{-}$ & $\rightarrow$ & $6^{-}$ & 653 \\
\hline $590.3(3)$ & 1.1 & & & 0 & $19^{+}$ & $\rightarrow$ & $18^{+}$ & 4547 \\
\hline $592.2(3)$ & 0.4 & & & 7 & $16^{-}$ & $\rightarrow$ & $15^{-}$ & 3545 \\
\hline $592.6(3)$ & 0.9 & & & 5 & $24^{-}$ & $\rightarrow$ & $23^{-}$ & 6787 \\
\hline 594.1(2) & 11.9 & & & 1 & $12^{+}$ & $\rightarrow$ & $10^{+}$ & 1273 \\
\hline $599.0(3)$ & 0.7 & & & 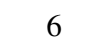 & $17^{-}$ & $\rightarrow$ & $15^{-}$ & 3736 \\
\hline 607.1(3) & 0.7 & & & 2 & $13^{+}$ & $\rightarrow$ & $11^{+}$ & 1911 \\
\hline $608.9(4)$ & 0.4 & & & 7 & $17^{-}$ & $\rightarrow$ & $15^{-}$ & 3861 \\
\hline $610.6(2)$ & 4.6 & $0.99(8)$ & $E 2$ & 4 & $13^{+}$ & $\rightarrow$ & $11^{+}$ & 2161 \\
\hline 612.1(4) & 0.4 & & & - & $25^{-}$ & $\rightarrow$ & $24^{-}$ & 7399 \\
\hline $613.5(3)$ & 0.8 & & & 6 & $18^{-}$ & $\rightarrow$ & $17^{-}$ & 4096 \\
\hline 614.1(4) & 0.2 & $0.36(22)$ & D & 12 & $7^{-}$ & $\rightarrow$ & $6^{-}$ & 82 \\
\hline $617.6(3)$ & 0.3 & & & 4 & $11^{+}$ & $\rightarrow$ & $9^{+}$ & 551 \\
\hline $620.4(2)$ & 9.6 & $0.83(27)^{\mathrm{c}}$ & D & 0 & $12^{+}$ & $\rightarrow$ & $11^{+}$ & 1587 \\
\hline $624.8(2)$ & 6.1 & & & 0 & $11^{+}$ & $\rightarrow$ & $10^{+}$ & 1304 \\
\hline $626.6(3)$ & 0.6 & & & 3 & $10^{+}$ & $\rightarrow$ & $10^{+}$ & 1306 \\
\hline $629.6(2)$ & 1.6 & & & 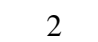 & $10^{+}$ & $\rightarrow$ & $9^{+}$ & 1138 \\
\hline $633.0(3)$ & 2.5 & & & 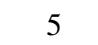 & $18^{-}$ & $\rightarrow$ & $16^{-}$ & 3826 \\
\hline 633.3(2) & 4.2 & $0.99(9)$ & $E 2$ & 11 & $11^{-}$ & $\rightarrow$ & $9^{-}$ & 1719 \\
\hline $633.7(3)$ & 0.8 & & & 11 & $12^{-}$ & $\rightarrow$ & $10^{-}$ & 2101 \\
\hline $637.9(2)$ & 3.2 & $0.56(32)$ & D & 2 & $13^{+}$ & $\rightarrow$ & $12^{+}$ & 1911 \\
\hline $642.9(3)$ & 0.8 & & & 9 & $9^{-}$ & $\rightarrow$ & $7^{-}$ & 1026 \\
\hline 644.9 & 3.5 & $0.93(8)$ & $E 2$ & 4 & $13^{+}$ & $\rightarrow$ & $11^{+}$ & 2161 \\
\hline $646.0(4)$ & 0.1 & & & 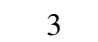 & $12^{+}$ & $\rightarrow$ & $10^{+}$ & 1784 \\
\hline $646.3(2)$ & 3.5 & & & 13 & $11^{-}$ & $\rightarrow$ & $9^{-}$ & 1779 \\
\hline $656.5(4)$ & 0.2 & & & 7 & $18^{-}$ & $\rightarrow$ & $16^{-}$ & 4201 \\
\hline $660.9(3)$ & 0.2 & & & 2 & $14^{+}$ & $\rightarrow$ & $12^{+}$ & 2248 \\
\hline $662.5(2)$ & 0.9 & & & $J$ & $14^{-}$ & $\rightarrow$ & $14^{+}$ & 2748 \\
\hline $669.3(3)$ & 0.4 & & & 6 & $16^{-}$ & $\rightarrow$ & $14^{-}$ & 3417 \\
\hline 677.7(4) & 0.6 & & & 11 & $13^{-}$ & $\rightarrow$ & $11^{-}$ & 2457 \\
\hline $678.9(3)$ & 0.5 & & & 6 & $18^{-}$ & $\rightarrow$ & $16^{-}$ & 4096 \\
\hline $680.0(2)$ & 0.7 & & & 6 & $15^{-}$ & $\rightarrow$ & $13^{-}$ & 3137 \\
\hline $680.6(2)$ & 1.9 & $2.17(38)^{\mathrm{c}}$ & $E 2$ & . & $10^{-}$ & $\rightarrow$ & $8^{-}$ & 1333 \\
\hline $680.8(4)$ & 0.4 & & & 6 & $19^{-}$ & $\rightarrow$ & $18^{-}$ & 4507 \\
\hline $682.9(3)$ & 3.8 & & & 10 & $10^{-}$ & $\rightarrow$ & $8^{-}$ & 1233 \\
\hline $683.6(4)$ & 0.8 & & & 11 & $7^{-}$ & $\rightarrow$ & $6^{-}$ & 751 \\
\hline 690.0 & 0.7 & $1.27(49)$ & $E 2$ & 13 & $12^{-}$ & $\rightarrow$ & $10^{-}$ & 2158 \\
\hline 6 & 0.7 & & & 2 & $21^{+}$ & $\rightarrow$ & $20^{+}$ & 5718 \\
\hline $702.1(2)$ & 0.5 & & & 5 & $14^{-}$ & $\rightarrow$ & $12^{-}$ & 2748 \\
\hline 702.1(3) & 2.4 & & & 3 & $14^{+}$ & $\rightarrow$ & $13^{+}$ & 2392 \\
\hline & 6.1 & 1. & $E 2$ & 10 & $11^{-}$ & $\rightarrow$ & $9^{-}$ & 1573 \\
\hline $704.2(2)$ & 2.1 & & & 5 & $15^{-}$ & $\rightarrow$ & $14^{+}$ & 2953 \\
\hline
\end{tabular}


TABLE I. (Continued.)

\begin{tabular}{|c|c|c|c|c|c|c|c|}
\hline$E_{\gamma}(\mathrm{keV})$ & $I_{\gamma}$ & $R_{\mathrm{DCO}}$ & Mult. & Band $_{i}$ & $I_{i}^{\pi}$ & $\rightarrow I_{f}^{\pi}$ & $E_{i}(\mathrm{keV})$ \\
\hline $704.7(6)$ & 0.8 & & & 3 & $17^{+}$ & $\rightarrow 16^{+}$ & 3722 \\
\hline $706.3(4)$ & 0.4 & & & 2 & $15^{+}$ & $\rightarrow 13^{+}$ & 2617 \\
\hline $714.8(3)$ & 2.3 & $2.31(40)^{\mathrm{c}}$ & $E 2$ & 11 & $12^{-}$ & $\rightarrow 10^{-}$ & 2101 \\
\hline $716.2(5)$ & 0.7 & & & 6 & $20^{-}$ & $\rightarrow 19^{-}$ & 4928 \\
\hline $718.5(3)$ & 0.9 & & & 7 & $18^{-}$ & $\rightarrow 17^{-}$ & 4201 \\
\hline $723.1(2)$ & 8.1 & & & 1 & $13^{+}$ & $\rightarrow 11^{+}$ & 1690 \\
\hline $724.2(4)$ & 0.5 & & & 2 & $10^{+}$ & $\rightarrow 8^{+}$ & 1138 \\
\hline $729.1(3)$ & 2.6 & $1.49(69)$ & $E 2$ & 5 & $19^{-}$ & $\rightarrow 17^{-}$ & 4212 \\
\hline $729.5(3)$ & 0.4 & & & $l s$ & $\left(7^{-}\right)$ & $\rightarrow 6^{-}$ & 797 \\
\hline $730.2(3)$ & 0.6 & & & 3 & $13^{+}$ & $\rightarrow 11^{+}$ & 2034 \\
\hline $737.0(2)$ & 0.8 & $0.93(28)$ & $E 2$ & 8 & $19^{-}$ & $\rightarrow 17^{-}$ & 4598 \\
\hline $738.4(3)$ & 3.6 & $0.95(11)$ & $E 2$ & 11 & $13^{-}$ & $\rightarrow 11^{-}$ & 2457 \\
\hline $742.3(3)$ & 1.9 & $1.02(35)$ & $E 2$ & 11 & $14^{-}$ & $\rightarrow 12^{-}$ & 2844 \\
\hline $744.2(7)$ & 0.3 & & & 3 & $18^{+}$ & $\rightarrow 17^{+}$ & 4233 \\
\hline $745.7(5)$ & 0.3 & & & 3 & $15^{+}$ & $\rightarrow 14^{+}$ & 2831 \\
\hline $748.4(3)$ & 0.4 & & & 6 & $14^{-}$ & $\rightarrow 13^{+}$ & 2910 \\
\hline $753.9(4)$ & 0.4 & & & 13 & $11^{-}$ & $\rightarrow 9^{-}$ & 1779 \\
\hline $758.2(3)$ & 0.3 & & & 3 & $9^{+}$ & $\rightarrow 8^{+}$ & 1172 \\
\hline $760.5(3)$ & 1.5 & & & 3 & $13^{+}$ & $\rightarrow 12^{+}$ & 2034 \\
\hline $763.9(4)$ & 0.2 & & & 5 & $13^{-}$ & $\rightarrow 12^{+}$ & 2548 \\
\hline $767.4(2)$ & 8.1 & $1.06(13)$ & $E 2$ & 4 & $15^{+}$ & $\rightarrow 13^{+}$ & 2929 \\
\hline $768.2(2)$ & 2.5 & & & 5 & $13^{-}$ & $\rightarrow 11^{-}$ & 2548 \\
\hline $769.3(3)$ & 1.3 & & & 2 & $16^{+}$ & $\rightarrow 14^{+}$ & 3017 \\
\hline $770.4(3)$ & 0.6 & $2.66(52)^{\mathrm{c}}$ & $E 2$ & 6 & $19^{-}$ & $\rightarrow 17^{-}$ & 4507 \\
\hline $771.4(3)$ & 0.5 & & & 13 & $12^{-}$ & $\rightarrow 10^{-}$ & 2158 \\
\hline $783.6(5)$ & 0.3 & & & 6 & $17^{-}$ & $\rightarrow 15^{-}$ & 3736 \\
\hline 783.7(3) & 0.8 & & & 7 & $18^{-}$ & $\rightarrow 16^{-}$ & 4201 \\
\hline 783.7(5) & 0.3 & & & 6 & $21^{-}$ & $\rightarrow 20^{-}$ & 5434 \\
\hline $785.3(4)$ & 0.2 & & & 6 & $22^{-}$ & $\rightarrow 21^{-}$ & 5912 \\
\hline $795.8(2)$ & 2.0 & & & 2 & $11^{+}$ & $\rightarrow 9^{+}$ & 1304 \\
\hline $797.0(3)$ & 0.5 & & & 3 & $15^{+}$ & $\rightarrow 13^{+}$ & 2831 \\
\hline $797.6(4)$ & 0.3 & & & 11 & $14^{-}$ & $\rightarrow 12^{-}$ & 2844 \\
\hline $797.9(3)$ & 0.2 & & & 3 & $9^{+}$ & $\rightarrow 7^{+}$ & 1172 \\
\hline 799.2(4) & 1.2 & & & 9 & $12^{-}$ & $\rightarrow 10^{-}$ & 2133 \\
\hline $804.6(3)$ & 0.8 & & & 3 & $14^{+}$ & $\rightarrow 12^{+}$ & 2392 \\
\hline $807.0(2)$ & 5.8 & & & 10 & $13^{-}$ & $\rightarrow 11^{-}$ & 2380 \\
\hline $808.5(4)$ & 0.3 & & & 6 & $14^{-}$ & $\rightarrow 12^{-}$ & 2910 \\
\hline $812.2(2)$ & 14.6 & & & 1 & $14^{+}$ & $\rightarrow 12^{+}$ & 2085 \\
\hline $812.9(3)$ & 3.3 & & & 10 & $12^{-}$ & $\rightarrow 10^{-}$ & 2046 \\
\hline $814.6(4)$ & 0.4 & & & 11 & $8^{-}$ & $\rightarrow 6^{-}$ & 882 \\
\hline $816.8(2)$ & 1.8 & & & 3 & $12^{+}$ & $\rightarrow 11^{+}$ & 1784 \\
\hline $822.5(2)$ & 4.6 & $1.00(13)$ & $E 2$ & 4 & $21^{+}$ & $\rightarrow 19^{+}$ & 5444 \\
\hline $822.6(2)$ & 1.5 & $1.44(57)$ & $E 2$ & 13 & $13^{-}$ & $\rightarrow 11^{-}$ & 2602 \\
\hline $824.3(3)$ & 2.3 & & & 5 & $20^{-}$ & $\rightarrow 18^{-}$ & 4650 \\
\hline $824.5(3)$ & 0.2 & & & 6 & $14^{-}$ & $\rightarrow 14^{+}$ & 2910 \\
\hline $825.3(4)$ & 0.4 & & & 8 & $21^{-}$ & $\rightarrow 19^{-}$ & 5459 \\
\hline $829.6(6)$ & 0.3 & & & 6 & $23^{-}$ & $\rightarrow 22^{-}$ & 6474 \\
\hline $832.2(4)$ & 0.5 & & & 6 & $20^{-}$ & $\rightarrow 18^{-}$ & 4928 \\
\hline $836.8(2)$ & 3.1 & & & 5 & $14^{-}$ & $\rightarrow 13^{+}$ & 2748 \\
\hline $837.0(3)$ & 1.9 & $0.60(10)$ & $\mathrm{D}$ & 3 & $11^{+}$ & $\rightarrow 10^{+}$ & 1516 \\
\hline $844.6(4)$ & 7.3 & 1.08(9) & $E 2$ & 4 & $19^{+}$ & $\rightarrow 17^{+}$ & 4622 \\
\hline $846.6(4)$ & 0.4 & $1.93(87)^{\mathrm{c}}$ & $E 2$ & 11 & $15^{-}$ & $\rightarrow 13^{-}$ & 3304 \\
\hline $848.3(4)$ & 8.1 & $0.94(13)$ & $E 2$ & 4 & $17^{+}$ & $\rightarrow 15^{+}$ & 3777 \\
\hline $857.6(2)$ & 4.6 & $1.40(52)$ & $\Delta I=0$ & 5 & $13^{-}$ & $\rightarrow 13^{+}$ & 2548 \\
\hline $858.1(4)$ & 0.4 & & & 7 & $20^{-}$ & $\rightarrow 18^{-}$ & 5059 \\
\hline $860.9(3)$ & 1.7 & $1.68(37)^{\mathrm{c}}$ & $E 2$ & 8 & $21^{-}$ & $\rightarrow 19^{-}$ & 5459 \\
\hline $861.5(5)$ & 0.5 & $1.69(33)^{\mathrm{c}}$ & $E 2$ & 8 & $19^{-}$ & $\rightarrow 17^{-}$ & 4598 \\
\hline $871.7(2)$ & 1.5 & & & 4 & $11^{+}$ & $\rightarrow 10^{+}$ & 1551 \\
\hline
\end{tabular}

TABLE I. (Continued.)

\begin{tabular}{|c|c|c|c|c|c|c|c|c|}
\hline$E_{\gamma}(\mathrm{keV})$ & $I_{\gamma}$ & $R_{\mathrm{DCO}}$ & Mult. & $\mathrm{Band}_{i}$ & $I_{i}^{\pi}$ & $\rightarrow$ & $I_{f}^{\pi}$ & $E_{i}(\mathrm{keV})$ \\
\hline $872.1(3)$ & 1.4 & & & 2 & $17^{+}$ & $\rightarrow$ & $15^{+}$ & 3489 \\
\hline $872.2(3)$ & 1.3 & $0.93(15)$ & $E 2$ & 7 & $15^{-}$ & $\rightarrow$ & $13^{-}$ & 3252 \\
\hline $874.1(4)$ & 0.5 & & & 9 & $14^{-}$ & $\rightarrow$ & $12^{-}$ & 3007 \\
\hline $876.4(5)$ & 0.2 & & & 6 & $14^{-}$ & $\rightarrow$ & $13^{+}$ & 2910 \\
\hline $882.4(2)$ & 3.5 & & & 4 & $23^{+}$ & $\rightarrow$ & $21^{+}$ & 6327 \\
\hline $883.1(2)$ & 0.8 & & & 13 & $13^{-}$ & $\rightarrow$ & $11^{-}$ & 2602 \\
\hline $884.3(3)$ & 0.7 & & & 3 & $16^{+}$ & $\rightarrow$ & $14^{+}$ & 3276 \\
\hline $890.5(5)$ & 0.2 & & & 3 & $17^{+}$ & $\rightarrow$ & $15^{+}$ & 3722 \\
\hline $891.3(2)$ & 6.9 & $0.95(11)$ & $E 2$ & 1 & $15^{+}$ & $\rightarrow$ & $13^{+}$ & 2581 \\
\hline $892.0(5)$ & 0.2 & & & 3 & $10^{+}$ & $\rightarrow$ & $8^{+}$ & 1306 \\
\hline $899.3(6)$ & 0.2 & & & 7 & $21^{-}$ & $\rightarrow$ & $19^{-}$ & 5533 \\
\hline $906.5(3)$ & 1.1 & $0.81(26)$ & $E 2$ & 10 & $15^{-}$ & $\rightarrow$ & $13^{-}$ & 3286 \\
\hline $907.9(2)$ & 1.7 & & & 2 & $12^{+}$ & $\rightarrow$ & $10^{+}$ & 1587 \\
\hline $908.4(2)$ & 1.0 & & & 2 & $17^{+}$ & $\rightarrow$ & $15^{+}$ & 3489 \\
\hline $914.1(3)$ & 1.2 & & & 1 & $22^{+}$ & $\rightarrow$ & $20^{+}$ & 6082 \\
\hline $914.7(3)$ & 2.3 & & & 5 & $21^{-}$ & $\rightarrow$ & $19^{-}$ & 5126 \\
\hline $916.3(4)$ & 0.8 & & & 10 & $14^{-}$ & $\rightarrow$ & $12^{-}$ & 2962 \\
\hline $920.1(3)$ & 0.8 & & & 3 & $15^{+}$ & $\rightarrow$ & $13^{+}$ & 2831 \\
\hline $923.7(5)$ & 0.2 & & & 11 & $15^{-}$ & $\rightarrow$ & $13^{-}$ & 3304 \\
\hline $927.4(4)$ & 0.6 & & & 7 & $16^{-}$ & $\rightarrow$ & $15^{+}$ & 3545 \\
\hline $927.6(2)$ & 1.9 & & & 2 & $15^{+}$ & $\rightarrow$ & $13^{+}$ & 2617 \\
\hline $928.1(5)$ & 0.4 & & & 6 & $21^{-}$ & $\rightarrow$ & $19^{-}$ & 5434 \\
\hline $930.6(4)$ & 0.2 & & & 5 & $12^{-}$ & $\rightarrow$ & $10^{-}$ & 2317 \\
\hline $931.7(2)$ & 2.4 & & & 2 & $16^{+}$ & $\rightarrow$ & $14^{+}$ & 3017 \\
\hline $939.0(3)$ & 2.5 & & & 2 & $18^{+}$ & $\rightarrow$ & $16^{+}$ & 3956 \\
\hline $940.1(3)$ & 1.5 & & & 1 & $21^{+}$ & $\rightarrow$ & $19^{+}$ & 5609 \\
\hline $945.1(4)$ & 1.5 & & & 2 & $13^{+}$ & $\rightarrow$ & $11^{+}$ & 1911 \\
\hline $949.5(4)$ & 0.8 & & & 1 & $23^{+}$ & $\rightarrow$ & $21^{+}$ & 6558 \\
\hline $956.8(4)$ & 0.3 & & & 3 & $18^{+}$ & $\rightarrow$ & $16^{+}$ & 4233 \\
\hline $963.1(4)$ & 0.3 & & & 7 & $20^{-}$ & $\rightarrow$ & $18^{-}$ & 5059 \\
\hline $964.2(3)$ & 1.7 & $1.07(31)$ & $E 2$ & 8 & $23^{-}$ & $\rightarrow$ & $21^{-}$ & 6423 \\
\hline $975.7(3)$ & 8.1 & $0.94(17)$ & $E 2$ & 1 & $16^{+}$ & $\rightarrow$ & $14^{+}$ & 3061 \\
\hline $977.3(2)$ & 2.6 & & & 4 & $25^{+}$ & $\rightarrow$ & $23^{+}$ & 7304 \\
\hline $983.7(6)$ & 0.6 & & & 6 & $22^{-}$ & $\rightarrow$ & $20^{-}$ & 5912 \\
\hline $990.2(4)$ & 0.6 & & & 1 & $17^{+}$ & $\rightarrow$ & $15^{+}$ & 3608 \\
\hline $990.8(5)$ & 0.2 & & & 3 & $19^{+}$ & $\rightarrow$ & $17^{+}$ & 4713 \\
\hline $994.0(3)$ & 1.8 & & & 5 & $22^{-}$ & $\rightarrow$ & $20^{-}$ & 5644 \\
\hline $1004.3(4)$ & 0.9 & & & 1 & $24^{+}$ & $\rightarrow$ & $22^{+}$ & 7086 \\
\hline $1015.2(5)$ & 0.1 & & & 5 & $11^{-}$ & $\rightarrow$ & $9^{-}$ & 2101 \\
\hline $1020.5(5)$ & 0.2 & & & 6 & $13^{-}$ & $\rightarrow$ & $13^{+}$ & 2710 \\
\hline $1026.5(4)$ & 0.3 & & & 7 & $21^{-}$ & $\rightarrow$ & $19^{-}$ & 5533 \\
\hline $1026.6(3)$ & 4.0 & & & 1 & $17^{+}$ & $\rightarrow$ & $15^{+}$ & 3608 \\
\hline $1028.2(5)$ & 0.9 & & & 3 & $16^{+}$ & $\rightarrow$ & $14^{+}$ & 3276 \\
\hline $1038.5(3)$ & 2.7 & & & 1 & $20^{+}$ & $\rightarrow$ & $18^{+}$ & 5168 \\
\hline $1039.5(5)$ & 0.2 & & & 6 & $23^{-}$ & $\rightarrow$ & $21^{-}$ & 6474 \\
\hline $1043.9(3)$ & 4.0 & & & 5 & $12^{-}$ & $\rightarrow$ & $12^{+}$ & 2317 \\
\hline $1051.6(5)$ & 0.7 & & & 1 & $25^{+}$ & $\rightarrow$ & $23^{+}$ & 7610 \\
\hline $1052.0(4)$ & 0.3 & & & 6 & $15^{-}$ & $\rightarrow$ & $14^{+}$ & 3137 \\
\hline $1057.2(4)$ & 1.6 & & & 2 & $19^{+}$ & $\rightarrow$ & $17^{+}$ & 4547 \\
\hline $1060.9(3)$ & 2.8 & & & 1 & $19^{+}$ & $\rightarrow$ & $17^{+}$ & 4669 \\
\hline $1062.7(4)$ & 1.0 & & & 8 & $25^{-}$ & $\rightarrow$ & $23^{-}$ & 7486 \\
\hline $1065.9(4)$ & 2.1 & & & 2 & $20^{+}$ & $\rightarrow$ & $18^{+}$ & 5022 \\
\hline $1067.8(4)$ & 1.5 & & & 5 & $23^{-}$ & $\rightarrow$ & $21^{-}$ & 6194 \\
\hline $1068.0(3)$ & 6.1 & & & 1 & $18^{+}$ & $\rightarrow$ & $16^{+}$ & 4129 \\
\hline $1074.7(3)$ & 1.7 & & & 4 & $27^{+}$ & $\rightarrow$ & $25^{+}$ & 8379 \\
\hline $1075.1(5)$ & 0.5 & & & 6 & $24^{-}$ & $\rightarrow$ & $22^{-}$ & 6987 \\
\hline $1112.4(5)$ & 0.7 & & & 1 & $18^{+}$ & $\rightarrow$ & $16^{+}$ & 4129 \\
\hline $1134.1(3)$ & 1.1 & & & 5 & $11^{-}$ & $\rightarrow$ & $11^{+}$ & 2101 \\
\hline
\end{tabular}


TABLE I. (Continued.)

\begin{tabular}{|c|c|c|c|c|c|c|c|c|}
\hline$E_{\gamma}(\mathrm{keV})$ & $I_{\gamma}$ & $R_{\mathrm{DCO}}$ & Mult. & Band $_{i}$ & $I_{i}^{\pi}$ & $\rightarrow$ & $I_{f}^{\pi}$ & $E_{i}(\mathrm{keV})$ \\
\hline $1142.7(5)$ & 1.1 & & & 5 & $24^{-}$ & $\rightarrow$ & $22^{-}$ & 6787 \\
\hline $1145.7(5)$ & 0.4 & & & 1 & $27^{+}$ & $\rightarrow$ & $25^{+}$ & 8756 \\
\hline $1149.0(4)$ & 0.7 & & & 8 & $27^{-}$ & $\rightarrow$ & $25^{-}$ & 8635 \\
\hline $1154.0(5)$ & 0.5 & & & 11 & $14^{-}$ & $\rightarrow$ & $13^{+}$ & 2844 \\
\hline $1166.8(5)$ & 1.2 & & & 4 & $29^{+}$ & $\rightarrow$ & $27^{+}$ & 9545 \\
\hline $1167.4(5)$ & 0.8 & & & 7 & $15^{-}$ & $\rightarrow$ & $14^{+}$ & 3252 \\
\hline $1170.6(5)$ & 0.7 & & & 2 & $21^{+}$ & $\rightarrow$ & $19^{+}$ & 5718 \\
\hline $1191.0(5)$ & 1.3 & & & 2 & $22^{+}$ & $\rightarrow$ & $20^{+}$ & 6213 \\
\hline $1191.2(6)$ & 0.6 & & & 2 & $23^{+}$ & $\rightarrow$ & $21^{+}$ & 6909 \\
\hline $1204.8(5)$ & 1.2 & & & 5 & $25^{-}$ & $\rightarrow$ & $23^{-}$ & 7399 \\
\hline $1240.5(5)$ & 0.4 & & & 8 & $29^{-}$ & $\rightarrow$ & $27^{-}$ & 9875 \\
\hline $1251.2(5)$ & 0.7 & & & 4 & $31^{+}$ & $\rightarrow$ & $29^{+}$ & 10797 \\
\hline $1260.8(7)$ & 0.7 & & & 5 & $26^{-}$ & $\rightarrow$ & $24^{-}$ & 8048 \\
\hline $1320.7(6)$ & 0.4 & & & 5 & $27^{-}$ & $\rightarrow$ & $25^{-}$ & 8720 \\
\hline $1328.7(5)$ & 0.1 & & & 13 & $13^{-}$ & $\rightarrow$ & $12^{+}$ & 2602 \\
\hline $1338.8(5)$ & 0.2 & & & 8 & $31^{-}$ & $\rightarrow$ & $29^{-}$ & 11214 \\
\hline $1349.3(7)$ & 0.2 & & & 5 & $28^{-}$ & $\rightarrow$ & $26^{-}$ & 9397 \\
\hline $1350.7(5)$ & 0.2 & & & 4 & $33^{+}$ & $\rightarrow$ & $31^{+}$ & 12147 \\
\hline $1407.5(7)$ & 0.2 & & & 5 & $29^{-}$ & $\rightarrow$ & $27^{-}$ & 10127 \\
\hline $1436.6(5)$ & 0.6 & & & 6 & $13^{-}$ & $\rightarrow$ & $12^{+}$ & 2710 \\
\hline
\end{tabular}

${ }^{2}$ Multipolarity was accepted from Ref. [43].

${ }^{\mathrm{b}}$ For multipolarity assignment see the text.

${ }^{\mathrm{c}}$ DCO ratio was determined using a dipole gate.

The spin and parity assignments of the levels in band 12 are based on the known $2^{-}$spin-parity of the ground state, the measured DCO ratios, and the internal conversion coefficients derived from the coincidence intensities. DCO ratios of all five low-energy transitions of band 12 indicate a stretched dipole character for these $\gamma$ rays. In order to determine the $M 1$ or $E 1$ character of these transitions, their relative coincidence intensities seen in the double-gated coincidence spectra, obtained by setting gates on the $404-152 \mathrm{keV}$ pair and the 404-131 keV pair, have been examined. In these spectra the relative total $(\gamma$-ray + conversion-electron) transition intensities for the 116-, 131-, 152-, 118-, and 164-keV transitions should be equal. By assuming an $M 1$ character for the 164-keV $\gamma$ ray, the relative total transition intensity in the cascade can be calculated, and the $\alpha_{\text {tot }}$ conversion coefficients of the other transitions can be derived by measuring their relative $\gamma$-ray intensities. This assumption can be justified: The 389- and the 451-keV transitions from the two lowest levels of band 13 have quadrupole character. As they have comparable intensities with the dipole transitions depopulating the same states, their multipolarity must be $E 2$. Thus the initial and the final states of the $164-\mathrm{keV}$ transition should have the same parity. The conversion coefficients are shown in Table II together with the theoretical conversion coefficients for $M 1$ and $E 1$ transitions.

It can be seen that the transitions have most likely $M 1$ character, with some probable $E 2$ admixture if the $164-\mathrm{keV}$ transition is $M 1$. In the last column of the table we show the conversion coefficients determined by assuming an $E 1$ character for the $164-\mathrm{keV}$ transition. In this case the experimental values lie halfway between the theoretical $E 1$ and $M 1$ values, which would imply an $E 1$ transition with a
TABLE II. Conversion coefficients of dipole transitions in band 12. The theoretical $\alpha_{M 1}$ and $\alpha_{E 1}$ values are taken from Ref. [44]. The $\alpha_{\exp }(1)$ values are derived with the assumption that the $164-\mathrm{keV}$ transition is $M 1$, while the $\alpha_{\text {exp }}(2)$ values are derived assuming an $E 1$ character for it.

\begin{tabular}{lcccc}
\hline \hline$E_{\gamma}(\mathrm{keV})$ & $\alpha_{M 1}$ & $\alpha_{E 1}$ & $\alpha_{\exp }(1)$ & $\alpha_{\exp }(2)$ \\
\hline 115.7 & 0.86 & 0.17 & $0.97(29)$ & $0.57(17)$ \\
117.9 & 0.81 & 0.16 & $0.86(26)$ & $0.49(15)$ \\
131.3 & 0.60 & 0.12 & $0.81(24)$ & $0.45(14)$ \\
152.5 & 0.39 & 0.08 & $0.65(19)$ & $0.33(10)$ \\
\hline \hline
\end{tabular}

strong $M 2$ admixture. This scenario is not expected for these transitions because a strong $M 2$ admixture would generate a significant lifetime, which was not observed. Thus the obtained conversion coefficients further confirm the assumption that the 164-keV transition has an $M 1$ character. As all the dipole transitions in band 12 have stretched $M 1$ character, all the levels in this band have negative parity and the spins of the consecutive levels must differ by $1 \hbar$. We assign increasing spin values to the levels with increasing excitation energy for the following reasons.

(i) The 11-min isomeric state is linked to the $5^{-}$and $7^{-}$ levels of band 12 by stretched dipole transitions. If both of these spins were smaller, then the spin of the isomeric state would be smaller than or equal to $4 \hbar$. In this case, however, there would be a possibility to decay to the ground state with a few orders of magnitude smaller lifetime than observed.

(ii) If only the $7^{-}$level had smaller spin value, and the spin of the 11-min isomeric state were $6 \hbar$, then many inter-band decays would show an "unnatural" pattern, which is not expected between high-spin rotational bands. For example, if the spin of the highest-energy level of band 12 was $5 \hbar$, then all the spins in band 11 , and consequently in band 5 , would be $2 \hbar$ less than is plotted in the level scheme. In this case the lowest levels of band 5 would decay to band 1 by strong $M 2$ transitions only, and not by $\Delta I=0 E 1$ transitions.

We note that the first excited low-spin state of ${ }^{134} \mathrm{Pr}$ reported in Ref. [45] has the same excitation energy within the experimental error as our $3^{-}$level in band 12 . Therefore, it is tempting to think that they are the same state. However, the spin assignment in Ref. [45] is $1^{-}$. Thus either the two states are different or the spin assignment is not correct in one of the cases.

The spin of the 11-min isomeric state is fixed by the 332and 614-keV stretched dipole transitions from band 12. It can only be $6 \hbar$, which is in agreement with the value suggested tentatively in Ref. [43]. The parities of the bands in Fig. 2 are assigned by assuming that the quadrupole transitions which compete with dipole transitions from the same state are E2 transitions. Using this argument all the bands in Fig. 2 are proved to have negative parity. For the 11-min isomeric state it is in good agreement with the tentative parity assignment in Ref. [43]. The spins are assigned on the basis of the measured DCO ratios by assuming an increasing spin with increasing excitation energy within the bands. 


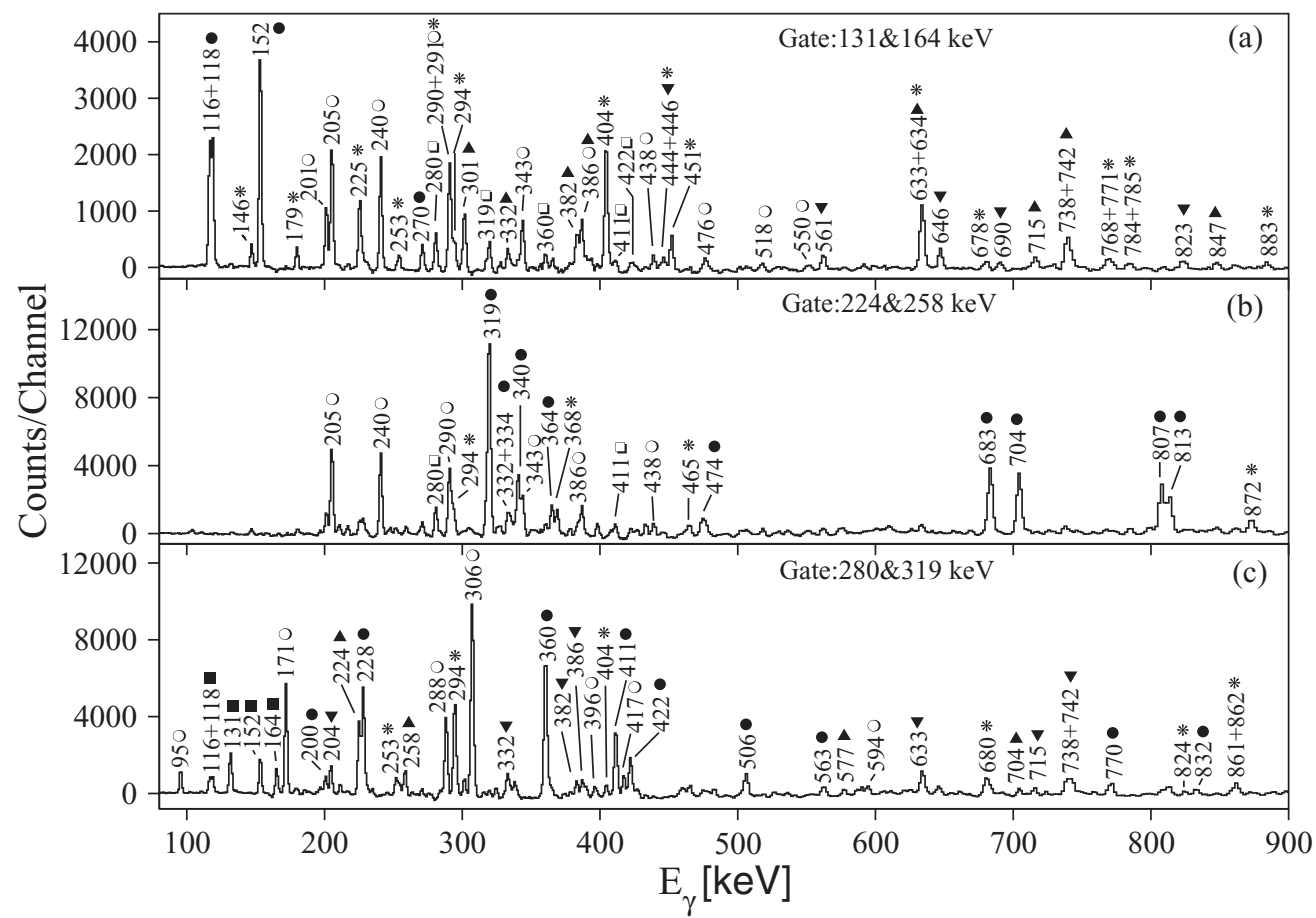

FIG. 1. Typical $\gamma \gamma \gamma$-coincidence spectra obtained in the present work. In panel (a) the double gate was set on band 12 transitions. Peaks labeled with full circles, full upward triangles, and full downward triangles belong to bands 12, 11, and 13, respectively, while peaks labeled with open circles and open squares belong to bands 5 and 6, respectively. In panel (b) the double gate was set on band 10 transitions. Peaks labeled with full circles, open circles, and open squares belong to bands 10, 5, and 6, respectively. In panel (c) the double gate was set on band 6 transitions. Peaks labeled with full circles, full squares, full upward triangle, and full downward triangles belong to bands $6,12,10$, and 11, respectively, while peaks labeled with open circles belong to band 1. Peaks labeled with stars in all the panels belong to transitions between bands.

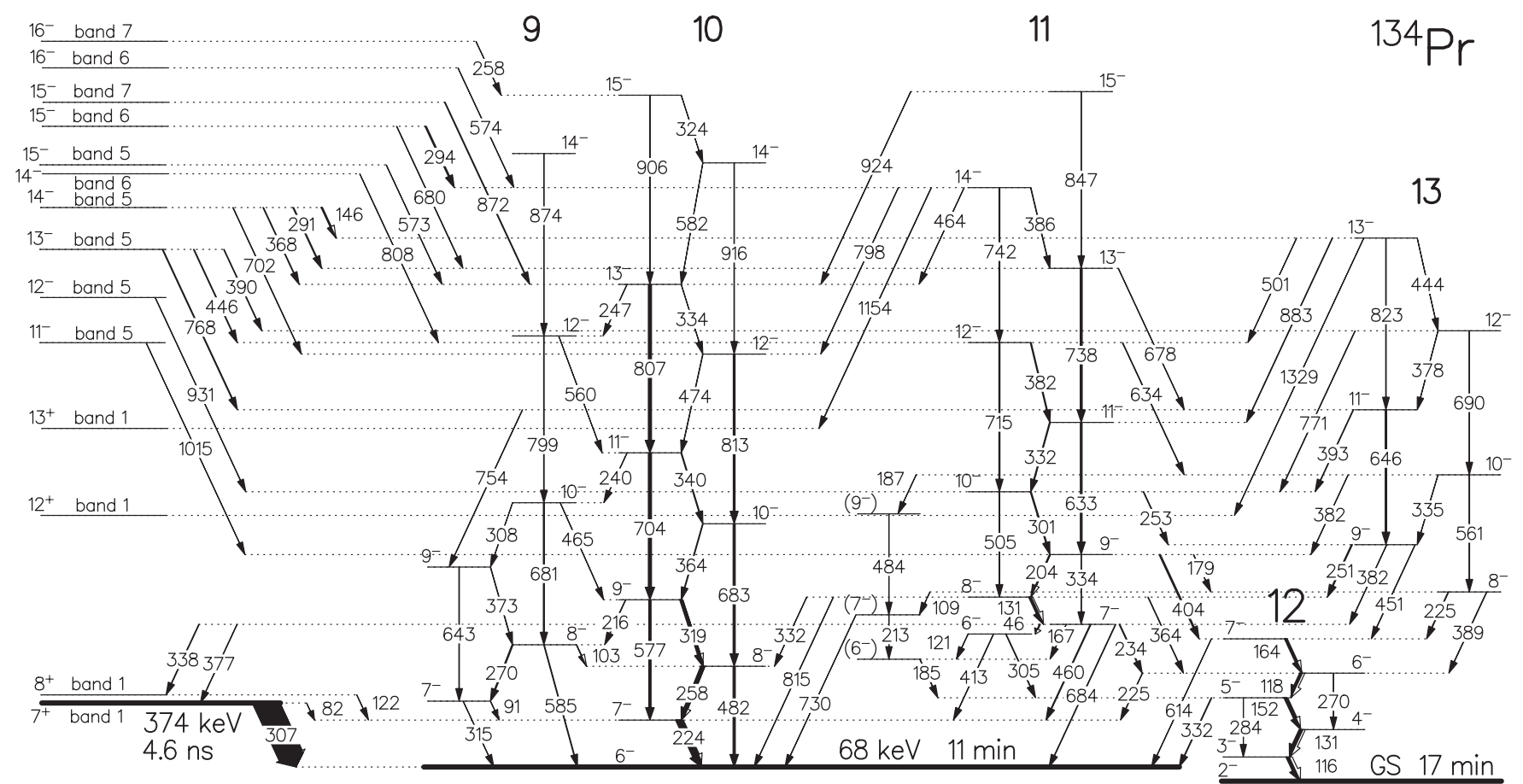

FIG. 2. Low- and medium-spin negative-parity bands of ${ }^{134} \mathrm{Pr}$ obtained in the present work. Linking transitions to the rest of the level scheme are plotted in the left side of the figure. The energies are given in $\mathrm{keV}$, while the widths of the transitions are proportional to their relative intensities. 


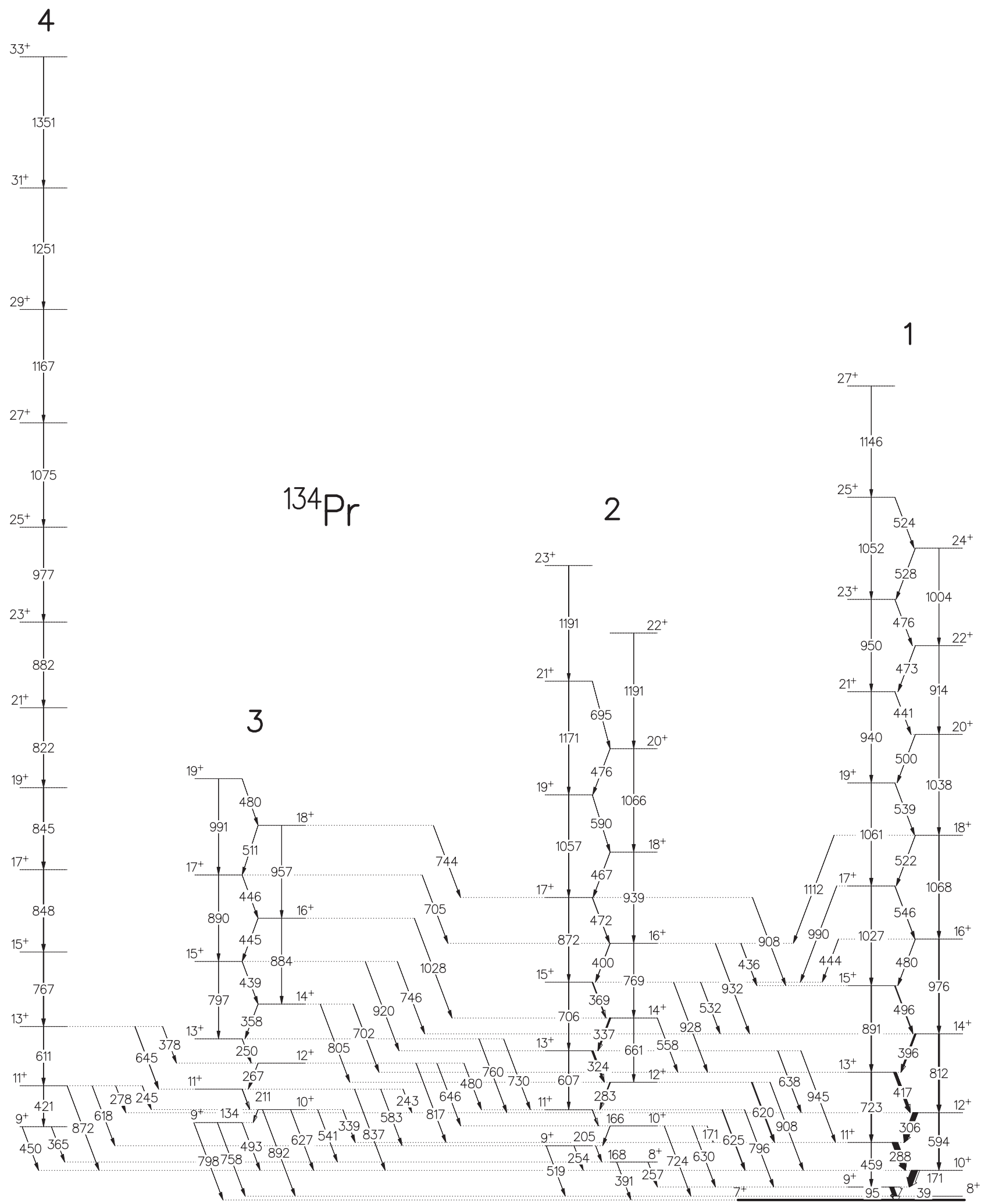

FIG. 3. Positive-parity bands of ${ }^{134} \mathrm{Pr}$ obtained in the present work. The energies are given in keV, while the widths of the transitions are proportional to their relative intensities.

Bands 11 and 13 have similar excitation energies and decay to each other through many dipole and quadrupole transitions, suggesting similar intrinsic structure. In addition, the properties of bands 10 and 9 are similar to each other, although there are fewer linking transitions and only dipoles are seen. There is a small level structure containing three levels 


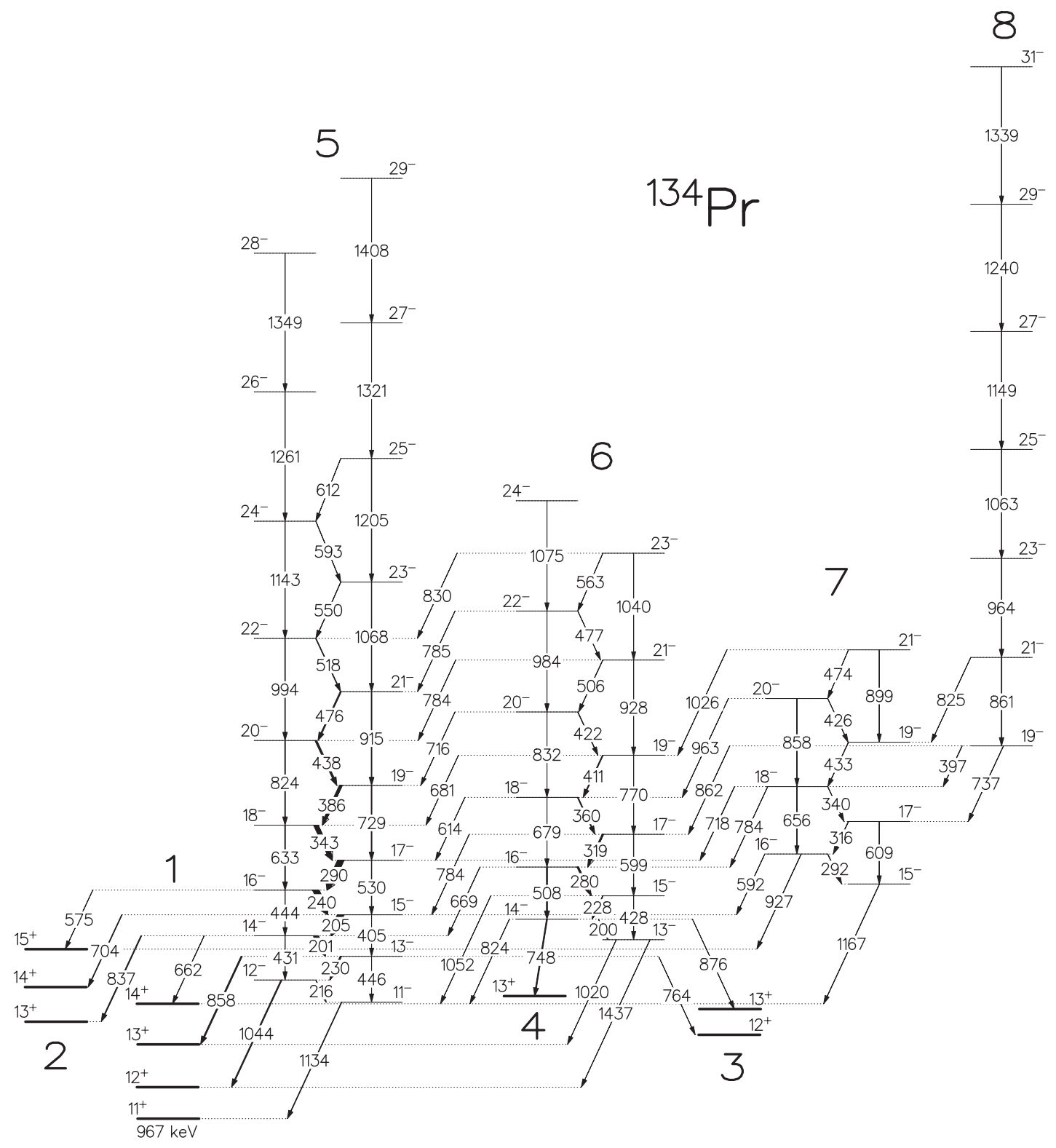

FIG. 4. High-spin negative-parity bands of ${ }^{134} \mathrm{Pr}$ obtained in the present work. In order to show the decays out to the positive-parity bands some levels of these bands are plotted by thick lines; however, the decays between these positive-parity levels are not plotted in this figure. The decays out to the negative-parity medium-spin bands are indicated in Fig. 2. The energies are given in keV, and the widths of the transitions are proportional to their relative intensities.

and linked mainly to bands 11,12 , and 13 . It is placed in between bands 10 and 11 in Fig. 2.

\section{B. Positive-parity bands}

The positive-parity bands observed in the present experiment are plotted in Fig. 3. These bands decay to the shortlived 4.6-ns isomeric state, which was previously reported in Ref. [43]. Spin-parity $7^{+}$has been tentatively assigned to this level in Ref. [43]. It was possible to confirm this assignment from the present experimental data. The $307-\mathrm{keV}$ transition from this level to the $6^{-} 11$-min isomeric state has a stretched dipole character according to the measured DCO ratio. Therefore the spin of the 4.6-ns level can be $7 \hbar$ or $5 \hbar$. In both cases its parity can be positive or negative. It is known from Ref. [43] that the 4.6-ns level is fed by a cascade of 39-, 95-, and 171-keV transitions, among which the first two have $M 1$ character. In the present experiment we observed an $82-\mathrm{keV}$ transition from the 4.6-ns level and a $122-\mathrm{keV}$ transition from the level depopulating by the $39-\mathrm{keV}$ transition. Both transitions go to the $7^{-}$state of band 10 , which decays to the $6^{-}$state by a $224-\mathrm{keV}$ transition (see Fig. 2). Examination of the relative coincidence intensities of the 95-, 82-, and $122-\mathrm{keV}$ transitions in the coincidence spectrum double-gated on the 171- and 224-keV transitions can assist in deriving spin and parity values for the 4.6-ns state. The coincidence 
TABLE III. Experimental coincidence $\gamma$-ray intensities derived from the spectrum double-gated by the 171- and 224-keV transitions, as well as calculated coincidence transition intensities assuming different spin-parity values for the 4.6-ns state. The theoretical conversion coefficient values are taken from Ref. [44].

\begin{tabular}{llllcc}
\hline \hline$E_{\gamma}(\mathrm{keV})$ & $I_{\gamma} \exp$ & $I_{t r}\left(7^{+}\right)$ & $I_{t r}\left(7^{-}\right)$ & $I_{t r}\left(5^{-}\right)$ & $I_{t r}\left(5^{+}\right)$ \\
\hline 94.6 & $13(4)$ & $33(10)$ & $33(10)$ & $33(10)$ & $33(10)$ \\
82.4 & $19(6)$ & $27(9)$ & $62(20)$ & $103(34)$ & $550(180)$ \\
121.9 & $13(4)$ & $15(5)$ & $23(7)$ & $23(7)$ & $15(5)$ \\
\hline \hline
\end{tabular}

$\gamma$-ray intensities can be obtained directly from the spectrum. The total transition intensities can be derived by taking into account the internal conversion coefficients, which depend on the multipolarities of the transitions and consequently on the spin and parity of the 4.6-ns state. In Table III we show the observed experimental $\gamma$-ray coincidence intensities for the 95-, 82-, and 122-keV transitions, as well as their calculated transition intensities for the four possible cases. In the case of the correct spin and parity the sum of transition intensities of the 82- and 122-keV transitions should be equal with the transition intensity of the $95-\mathrm{keV}$ transition. As is seen in the table, only the $7^{+}$spin-parity complies with this condition. Thus, the spin-parity of the 4.6-ns state is $7^{+}$, which is in good agreement with the value tentatively suggested in Ref. [43].

The spin values of the higher-energy levels in Fig. 3 are derived using the multipolarities of the depopulating transitions obtained from the measured DCO ratios, by assuming that in the case of stretched transitions the spins increase with increasing excitation energy within a band. These spin assignments are confirmed also by the many linking transitions between the different bands, for all of which the experimental DCO ratios are consistent with the spin differences of the levels they connect. The parities of the bands are fixed by the quadrupole transitions that compete with dipole transitions from the same level. These transitions are considered to have E2 character. This argument provides positive parity for all four bands in Fig. 3. Bands 1, 2, and 4 have been reported previously, while band 3 was derived first from the data of the present experiment. This band is linked by many dipole and quadrupole transitions to bands 1 and 2 .

\section{High-spin negative-parity bands}

The negative-parity high-spin bands observed for the present experiment are plotted in Fig. 4. Band 5 has been previously reported in Refs. $[3,28]$. The other bands in the figure are observed for the first time in our experiment. Bands 5,6 , and 7 decay both to the lower-energy parts of the positiveparity bands and to the medium-spin negative-parity bands. The experimental DCO ratios of these decay-out transitions, as well as those of the transitions within the bands and between the bands, unambiguously fix the spin and parity values of the levels. According to these assignments all the bands in Fig. 4 have negative parity. A unique feature of the decay out of band 5 is that it feeds band 1 only by $\Delta I=0$ dipole transitions and band 2 only by stretched dipole transitions. Band 6 is linked to band 5 by many stretched dipole transitions, while band 7 is linked to band 6 by many stretched quadrupole transitions.

\section{DISCUSSION}

In this section we attempt to assign intrinsic single-particle configurations to the bands in ${ }^{134} \mathrm{Pr}$. Measured $B(M 1) / B(E 2)$ ratios, alignments, band-crossing frequencies, bandhead spins, and the single-particle configurations in the neighboring nuclei, as well as the predictions of total Routhian surface (TRS) and tilted-axis cranking (TAC) calculations are used to determine the configurations.

According to the observed intrinsic configurations in ${ }^{132} \mathrm{Ce}$ and neighboring odd- $A$ nuclei, the active single-particle states in ${ }^{134} \operatorname{Pr}$ are the $[541] 3 / 2^{-},[411] 3 / 2^{+}$, and $[413] 5 / 2^{+}$proton states, as well as the $[514] 9 / 2^{-},[400] 1 / 2^{+},[660] 1 / 2^{+}$and $[404] 7 / 2^{+}$neutron states. Indeed, Ref. [46] reports on bands based on the rotation-aligned [541]3/2- Nilsson state of $h_{11 / 2}$ parentage, as well as bands based on the deformation-aligned [411]3/2 $2^{+}$, and [413]5/2 $2^{+}$states of $d_{5 / 2}$ and $g_{7 / 2}$ parentage, respectively, in ${ }^{133} \mathrm{Pr}$. Similarly, Ref. [47] reports on a band based on the [400]1/2 $2^{+}$state derived from the $s_{1 / 2}$ subshell, bands based on the deformation-aligned $[514] 9 / 2^{-}$state derived from the $h_{11 / 2}$ subshell, and a band based on the rotation-aligned [660]1/2 $2^{+}$state of $i_{13 / 2}$ parentage in ${ }^{133} \mathrm{Ce}$. Furthermore, previous experimental results on ${ }^{134} \mathrm{Pr}$ suggest the possible presence of the $[541] 1 / 2^{-}$or $[530] 1 / 2^{-}$intruder neutron orbitals [3], while the presence of the $[404] 7 / 2^{+}$ neutron orbital was reported in Ref. [48]. In the case of the odd-odd ${ }^{134} \mathrm{Pr}$ we expect bands that are based on the combinations of the above single-proton and single-neutron states.

\section{A. Analysis of the $B(M 1) / B(E 2)$ ratios}

In order to assist in the configuration assignments we have derived the experimental in-band $B[M 1 ; I \rightarrow(I-$ $1)] / B[E 2 ; I \rightarrow(I-2)]$ ratios for the $\Delta I=1$ bands using the expression

$$
\begin{aligned}
& \frac{B(M 1 ; I \rightarrow I-1)}{B(E 2 ; I \rightarrow I-2)} \\
& \quad=0.697 \frac{E_{\gamma}^{5}(E 2)}{E_{\gamma}^{3}(M 1)} \frac{I_{\gamma}(M 1)}{I_{\gamma}(E 2)} \frac{1}{1+\delta^{2}}\left(\frac{\mu_{N}}{e b}\right)^{2},
\end{aligned}
$$

where the energies of the $\gamma$ rays are given in $\mathrm{MeV}$. The $\delta$-multipole mixing ratios of the $\Delta I=1$ transitions were assumed to be small, and thus $\delta^{2}$ can be neglected. The experimental $B(M 1) / B(E 2)$ ratios were compared to calculated values obtained using a generalized expression of the geometrical model of Dönau and Frauendorf [49], formulated in Refs. [50,51]. In the calculation, the $K$ and $i_{x}$ values were approximated with constant values, and these are listed in Table IV together with the appropriate $g$ values taken from [52]. The rotational gyromagnetic factor was taken as $g_{R}=Z / A$, while the $Q_{0}$ electric quadrupole moments and the $\gamma$ shape parameters were derived from the nuclear shape predicted by the TRS calculations. As the results of the TRS calculations failed to reproduce satisfactorily 
TABLE IV. Parameters used for the calculation of $B(M 1) / B(E 2)$ ratios.

\begin{tabular}{lccc}
\hline \hline Configuration & $g$ factor & $K$ value & $i_{x}$ \\
\hline$\pi h_{11 / 2}$ & 1.33 & 1.5 & 4.5 \\
$\pi g_{7 / 2}$ & 0.75 & 2.5 & 1.5 \\
$\pi d_{5 / 2}$ & 1.40 & 1.5 & 1.5 \\
$\nu h_{11 / 2}$ & -0.18 & 4.5 & 2 \\
$\nu s_{1 / 2}$ & -1.27 & 0.5 & 0 \\
$\nu g_{7 / 2}$ & 0.3 & 3.5 & 1 \\
\hline \hline
\end{tabular}

the experimental results, the obtained shape parameters are ambiguous. Moreover, within the TRS there is no tilted axis cranking possible, which is expected in this nucleus. Nevertheless, it is expected that the calculated $B(M 1) / B(E 2)$ ratios still can serve as a guide in the configuration assignment, as there can be large differences (an order of magnitude or more) between the $B(M 1) / B(E 2)$ ratios of the different configurations. Because of this approximate treatment of the calculated values, the signature splitting was not taken into account in the calculations.

The experimental and calculated $B(M 1) / B(E 2)$ ratios are compared in Fig. 5. Although all the possible configurations were taken into account in the calculations, only the configurations with calculated $B(M 1) / B(E 2)$ ratios reasonably close to the experimental values are plotted. For the possible configurations not shown, the calculated values differ from the experimental ones by about an order of magnitude or more.

\section{B. Analysis of the alignments and Routhians}

In order to confirm and further specify the assigned configurations, we have also derived the experimental alignments $\left(i_{x}\right)$ and Routhians $\left(e^{\prime}\right)$ of the bands as they are defined in [53] and plotted them in Fig. 6. A rotational reference, with a variable moment of inertia $J_{\text {ref }}=J_{0}+\omega^{2} J_{1}$, has been subtracted with Harris parameters $J_{0}=12 \hbar^{2} \mathrm{MeV}^{-1}$ and $J_{1}=29 \hbar^{4} \mathrm{MeV}^{-3}$ (as in Ref. [3]). In the derivation we assumed a $K=6$ value for the bands $1,2,3,5,6,7,9,10,11$, and $13 . K=1$ was assumed for the doubly decoupled bands 4 and 8 , and $K=2$ for band 12 .

The experimental total Routhians $\left(E^{\prime}\right)$ and aligned total angular momenta $\left(I_{x}\right)$ are also compared to the TRS calculations based on the Woods-Saxon cranking formalism [54-56]. The labeling of the TRS orbitals is given in Table V. The compared values are plotted in Fig. 8 for the negative-parity twoquasiparticle bands. The Routhians are normalized by adding the same constant value to all the experimental configurations in a way that the predicted and experimental values for band 10 are consistent at $\hbar \omega \approx 0.25 \mathrm{MeV}$.

\section{Band configurations}

\section{The positive-parity bands}

The configuration and the possible chiral nature of bands 1 and 2 have been thoroughly discussed in several previous papers [1,4,9,23-27]. According to these results bands 1 and

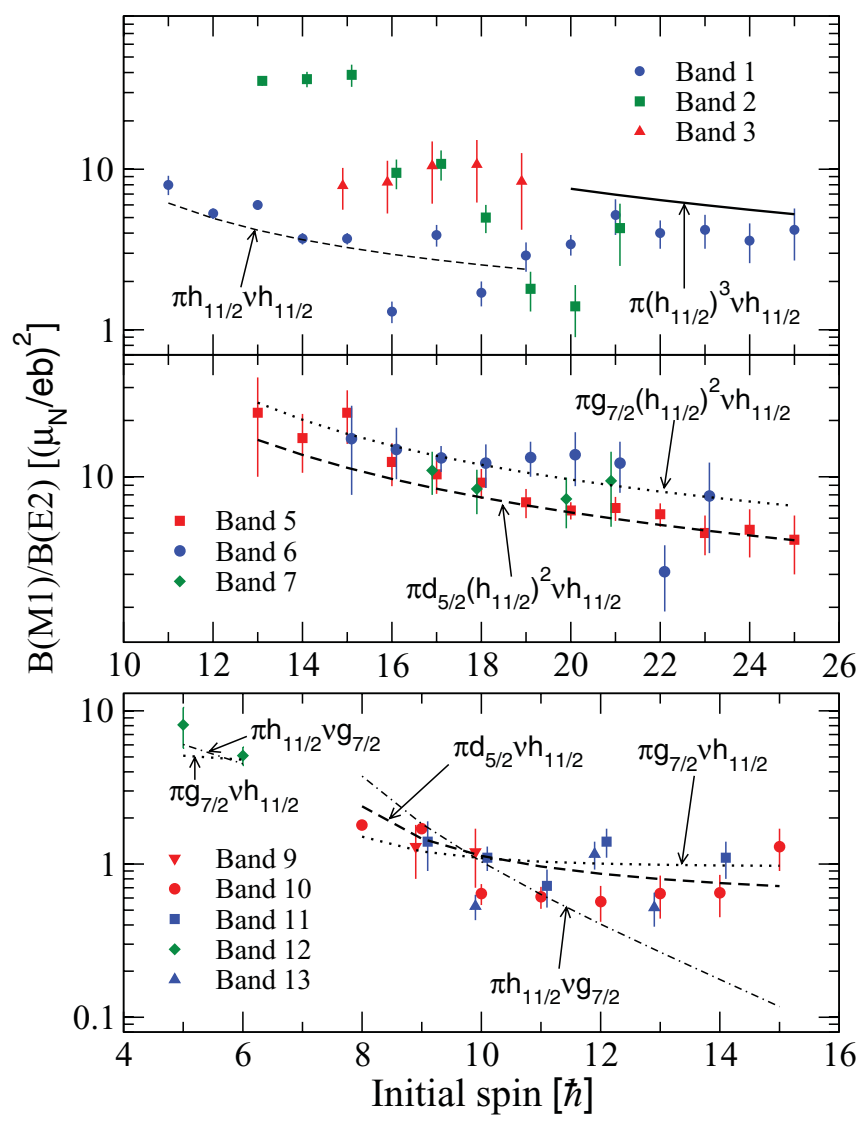

FIG. 5. (Color online) Experimental $B(M 1) / B(E 2)$ ratios of the ${ }^{134} \mathrm{Pr}$ bands obtained in the present work compared with calculated values assuming different configurations.

2 both have $\pi h_{11 / 2} v h_{11 / 2}$ configurations. In the present work, we confirm the previous tentative spin assignments; however, no new significant experimental data could be derived for these bands, which would affect the previous assignments and theoretical considerations. Thus we accept the previous interpretations.

Band 4 was discussed in Refs. [27,29,30] and has been suggested to correspond to the $\pi h_{11 / 2} v\left(f_{7 / 2}, h_{9 / 2}\right)$ configuration below the first alignment frequency. The $f_{7 / 2}$ and $h_{9 / 2}$ neutron orbitals, corresponding to the [530]1/2 and [541]1/2 Nilsson states, are strongly mixed in this region. Without

TABLE V. Labels used for the quasiproton (p) and quasineutron (n) states of parity $\pi$ and signature $\alpha$; $n$ denotes the $n$th state for a given set of $(\pi, \alpha)$ quantum numbers.

\begin{tabular}{lccllll}
\hline \hline \multicolumn{3}{c}{$\mathrm{p}$} & & & \multicolumn{3}{c}{$\mathrm{n}$} \\
\cline { 1 - 2 } \cline { 5 - 7 }$(\pi, \alpha)_{n}$ & Label & Shell model & & $(\pi, \alpha)_{n}$ & Label & Shell model \\
\hline$(+,+1 / 2)_{1}$ & $\mathrm{~A}$ & $d_{5 / 2}, g_{7 / 2}$ & & $(+,+1 / 2)_{1}$ & $\mathrm{a}$ & $g_{7 / 2}$ \\
$(+,-1 / 2)_{1}$ & $\mathrm{~B}$ & $d_{5 / 2}, g_{7 / 2}$ & & $(+,-1 / 2)_{1}$ & $\mathrm{~b}$ & $g_{7 / 2}$ \\
$(+,+1 / 2)_{2}$ & $\mathrm{C}$ & $d_{5 / 2}, g_{7 / 2}$ & & $(-,-1 / 2)_{1}$ & $\mathrm{e}$ & $h_{11 / 2}$ \\
$(+,-1 / 2)_{2}$ & $\mathrm{D}$ & $d_{5 / 2}, g_{7 / 2}$ & & $(-,+1 / 2)_{1}$ & $\mathrm{f}$ & $h_{11 / 2}$ \\
$(-,-1 / 2)_{1}$ & $\mathrm{E}$ & $h_{11 / 2}$ & & & \\
$(-,+1 / 2)_{1}$ & $\mathrm{~F}$ & $h_{11 / 2}$ & & & \\
$(-,-1 / 2)_{2}$ & $\mathrm{G}$ & $h_{11 / 2}$ & & & \\
\hline \hline
\end{tabular}



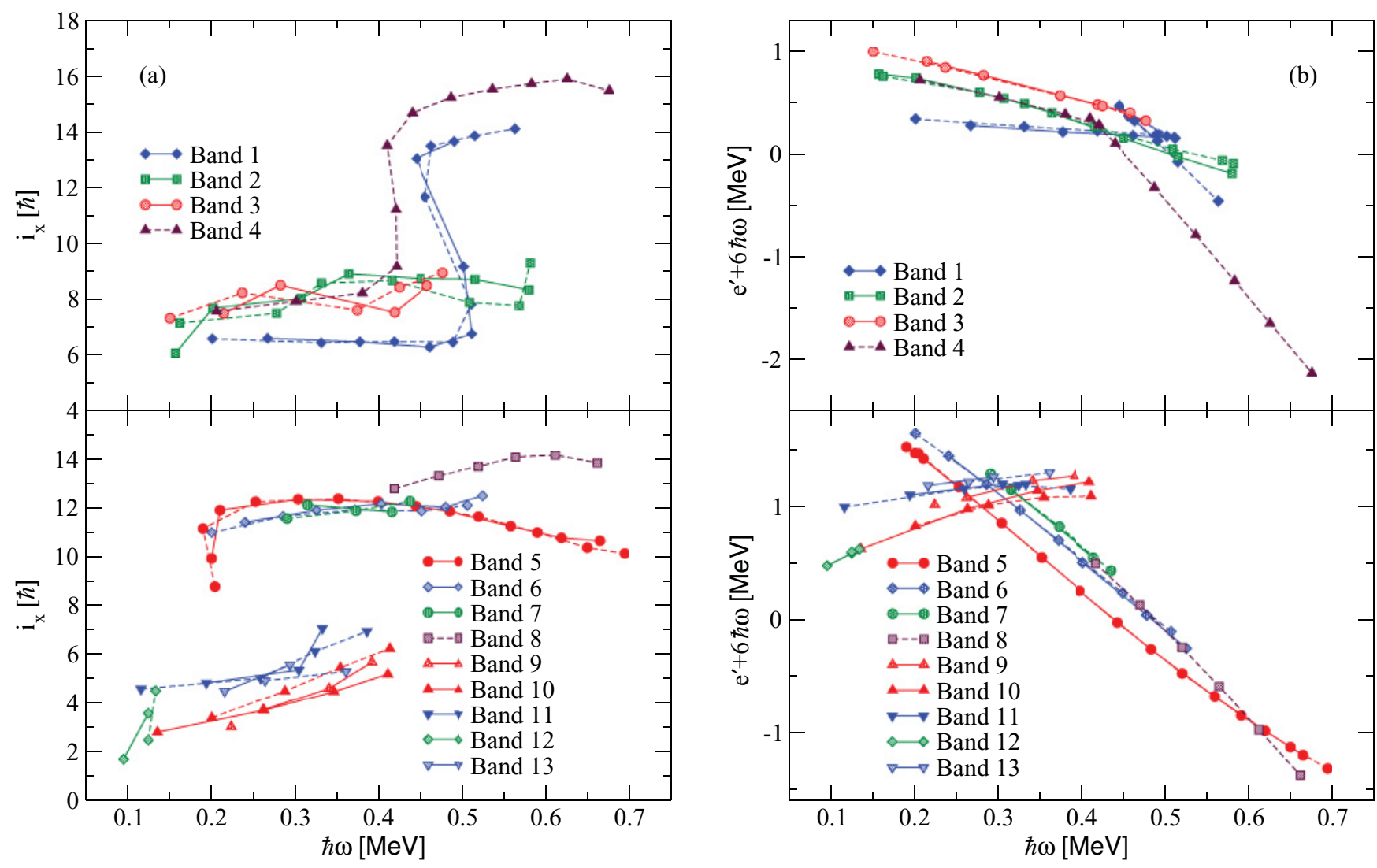

FIG. 6. (Color online) Alignments (a) and Routhians (b) of the bands observed in the present experiment as a function of the rotational frequency. Experimental points connected by solid (dashed) lines correspond to the even-spin (odd-spin) branch of the band.

mixing, the coupling of the [530]1/2 neutron Nilsson orbital with the $[541] 3 / 2$ proton Nilsson orbital would lead to a doubly decoupled band having odd-spin states, while the [541]1/2 neutron Nilsson state would generate an even-spin band. Petrache et al. [27] assigned odd spins to this band, and therefore a $\pi h_{11 / 2} \nu[530] 1 / 2$ configuration was suggested. On the other hand, Hauschild et al. [29] assigned even spins to the states in band 4 on the basis of the measured DCO ratios for the linking transitions between bands 4 and 1. Later Roberts et al. [43] increased the spin values of the band 1 states by 1 unit, which led again to odd-spin states in band 4. According to the present results, the states have odd spins. Therefore, most probably the main component of the configuration of band 4 is $\pi h_{11 / 2} v[530] 1 / 2$. This assignment is also in good agreement with the measured bandhead spin of $9 \hbar$, which is the sum of the single-particle spins expected for the rotation-aligned $f_{7 / 2}$ neutron and $h_{11 / 2}$ proton.

The alignment around $\hbar \omega=0.45 \mathrm{MeV}$ in bands 1 and 4 has been thought to correspond to the proton FG crossing in the previous assignments [27,29] on the basis of TRS calculations and the observed alignment gain. The predicted FG crossing frequency is about $\hbar \omega=0.5 \mathrm{MeV}$. The lower observed crossing frequency ( $\hbar \omega=0.41 \mathrm{MeV}$ ) for band 4 was attributed to a reduced pairing strength due to blocking of the first $\pi h_{11 / 2}$ proton crossing [29]. However, in this case the crossing frequency for band 1 would also be reduced. In the case of band 4 the neutron ef crossing is also possible, and it is expected to be characterized by a similar alignment gain to that of the proton FG crossing, however at a lower rotational frequency. The $B(M 1) / B(E 2)$ ratios observed in the present experiment for the upper part of band 1 confirm the FG crossing scenario for band 1, while the lower crossing frequency favors the neutron ef crossing scenario in the case of band 4 . We need to mention, however, that although both the ef and FG crossings are expected to occur in band 4, only one crossing is observed up to $\hbar \omega=0.65 \mathrm{MeV}$. The reason for this inconsistency is not clear. A possible explanation might be that due to the gamma softness of ${ }^{134} \mathrm{Pr}$ the ef crossing drives the nuclear shape to triaxial characterized by a negative gamma value, which pushes the FG crossing to higher rotational frequency.

Band 3 is newly identified in the present work. This $\Delta I=1$ band has positive parity, and it decays through several $M 1$ and $E 2$ transitions to bands 1 and 2. It has an alignment value of around $8 \hbar$, which corresponds to a two-quasiparticle configuration. These facts indicate that its configuration is $\pi h_{11 / 2} v h_{11 / 2}$, similar to the configuration of bands 1 and 2 . The observed $B(M 1) / B(E 2)$ ratios of band 3 are two to three times larger than the corresponding ratios of band 1 and are close to the $B(M 1) / B(E 2)$ ratios of band 2 near the $17 \hbar$ spin region. This suggests a similarity between bands 2 and 3, which might indicate that the two bands are chiral partners. In this context, it may be informative to inspect also the signature splittings of the three $\pi h_{11 / 2} v h_{11 / 2}$ bands. In the 


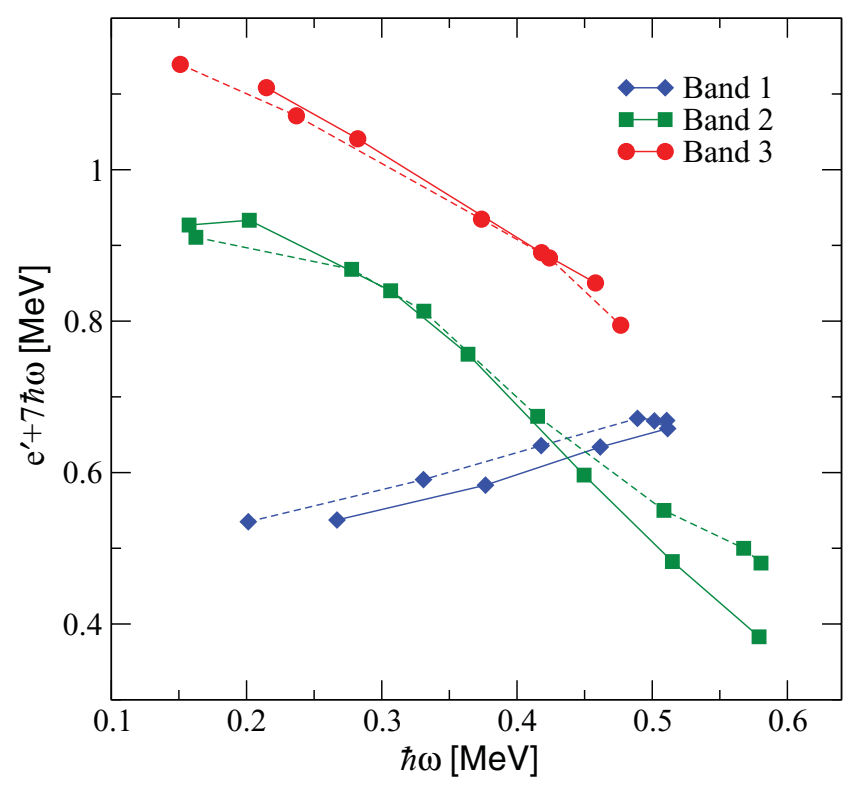

FIG. 7. (Color online) Observed signature splittings for the three positive-parity $\Delta I=1$ bands.

case of chiral geometry a small signature splitting is expected for the bands in the region of stable chirality. (Although the signature is not a good quantum number in the chiral case, we still use here the expression "signature splitting" for the observed odd-even staggering.) Thus, it is also a test for the chiral scenario. In Fig. 7, the Routhians for bands 1, 2, and 3 are plotted below the first alignment in band 1. Band 1 shows a constant $\sim 30-\mathrm{keV}$ signature splitting through the whole plotted frequency region, while the signature splittings of the two other bands are very small, being less than $10 \mathrm{keV}$ in the $\hbar \omega=0.3-0.4 \mathrm{MeV}$ frequency region. This fact might show that if there are chiral doublet bands among these three bands, the doublet is not bands 1 and 3, but rather bands 2 and 3 . This scenario seems to be also confirmed by the very similar alignment values for bands 2 and 3, while band 1 has a considerably smaller alignment value. Moreover, the signature splitting for band 1 is inverted. Low-spin signature inversion occurs systematically in this mass region for the $\pi h_{11 / 2} v h_{11 / 2}$ bands [57]. The exact cause is still not understood completely; however, it has been suggested that both the triaxial shape of the nucleus and the proton-neutron residual interaction play a significant role. It is expected when the nuclear shape is triaxial with a positive $\gamma$ value corresponding to the Lund convention [58]. This corresponds to the rotation around the small deformation axis instead of the medium axis, which would correspond to the chiral scenario. However, we stress again that the cause of the signature inversion is not well known yet, and thus we cannot draw strong conclusions on the shape of the nucleus only on the basis of the signature inversion.

\section{The negative-parity two-quasiparticle bands}

Bands 9-13 were not known before the present work. Their small alignment values in Fig. 6 indicate that they correspond to two-quasiparticle states. They are all $\Delta I=1$ bands with negative parity. Thus, they should correspond to either the $\pi[541] 3 / 2 \nu([400] 1 / 2$ or $[404] 7 / 2)$ or the $\pi([413] 5 / 2$ or $[411] 3 / 2) v[514] 9 / 2$ configurations.

It would be straightforward to think that these bands correspond to the lowest-energy first few negative-parity configurations predicted by the TRS calculations. In Fig. 8 we compared the experimental Routhians and aligned angular momenta of bands 9-13 with that of the low-energy negativeparity TRS configurations. The lowest experimental Routhians belong to band 12 at the lowest frequencies, and they belong to bands 9 and 10 at higher frequencies. It is the $(\mathrm{Ea}, \mathrm{Eb})$ TRS configuration pair that is predicted to have the lowest Routhians up to about $\hbar \omega=0.25 \mathrm{MeV}$. By taking into account that this configuration corresponds to a semidecoupled band, and given that no band similar to band 12 has been observed, band 12 could correspond to this configuration. The observed and calculated $B(M 1) / B(E 2)$ ratios are also in good agreement with this assignment. However, there are features which contradict this assumption. One is the signature splitting. Very small signature splitting is observed in band 12, while quite large signature splitting (around $100 \mathrm{keV}$ ) is expected for the (Ea,Eb) TRS configurations, although the predicted signature splitting depends strongly on the $\gamma$ deformation parameter and the nucleus is very $\gamma$ soft. A more serious discrepancy is related to the observed and predicted alignment values. At a rotational frequency of $\hbar \omega \approx 0.1 \mathrm{MeV}$ the predicted alignment value is around $6 \hbar$, reflecting the rotation-aligned nature of the $h_{11 / 2}$ proton, while the observed alignment is only around $3 \hbar$, suggesting a configuration without the rotation-aligned $h_{11 / 2}$ proton orbital.

Indeed, the bandhead of band 12 has a spin value of $2 \hbar$. Such a small bandhead spin cannot be built from a configuration that contains the rotation-aligned $[541] 3 / 2^{-}$ proton state. Thus, the possible configuration of this band is either $\pi[413] 5 / 2 \nu[514] 9 / 2$ or $\pi[411] 3 / 2 \nu[514] 9 / 2$, both of which contain deformation-aligned orbitals. According to the Gallagher-Moskowski rule [59], the bandhead spin of the first configuration would be $2 \hbar$, while the bandhead of the second configuration is expected to have $6 \hbar$ spin. Thus, it is a sensible assumption that band 12 corresponds to the $\pi[413] 5 / 2 v[514] 9 / 2$ configuration. The calculated $B(M 1) / B(E 2)$ ratios for the $\pi g_{7 / 2} \nu h_{11 / 2}$ configuration also reproduce the experimental values quite well.

Bands 9 and 10 are $\Delta I=1$ bands linked to each other by several $\gamma$ transitions, which indicates that they may correspond to two-quasiparticle configurations built up from deformationaligned proton and neutron orbitals. The situation is the same for bands 11 and 13. Thus, the probable configurations of these bands are $\pi[413] 5 / 2 \nu[514] 9 / 2$ or $\pi[411] 3 / 2 \nu[514] 9 / 2$. The calculated $B(M 1) / B(E 2)$ ratios for both the $\pi g_{7 / 2} \nu h_{11 / 2}$ and $\pi d_{5 / 2} \nu h_{11 / 2}$ configurations reproduce reasonably well the observed ratios of all four bands, as the experimental values of the different bands are very similar. Although the calculated $B(M 1) / B(E 2)$ ratio values for the $\pi h_{11 / 2} v g_{7 / 2}$ configuration are also relatively close to the experimental values, this configuration assignment is not that probable because the slope of the calculated curve is quite different from that of the experimental values as a function of spin. Moreover, this configuration contains the rotation-aligned (decoupled) 


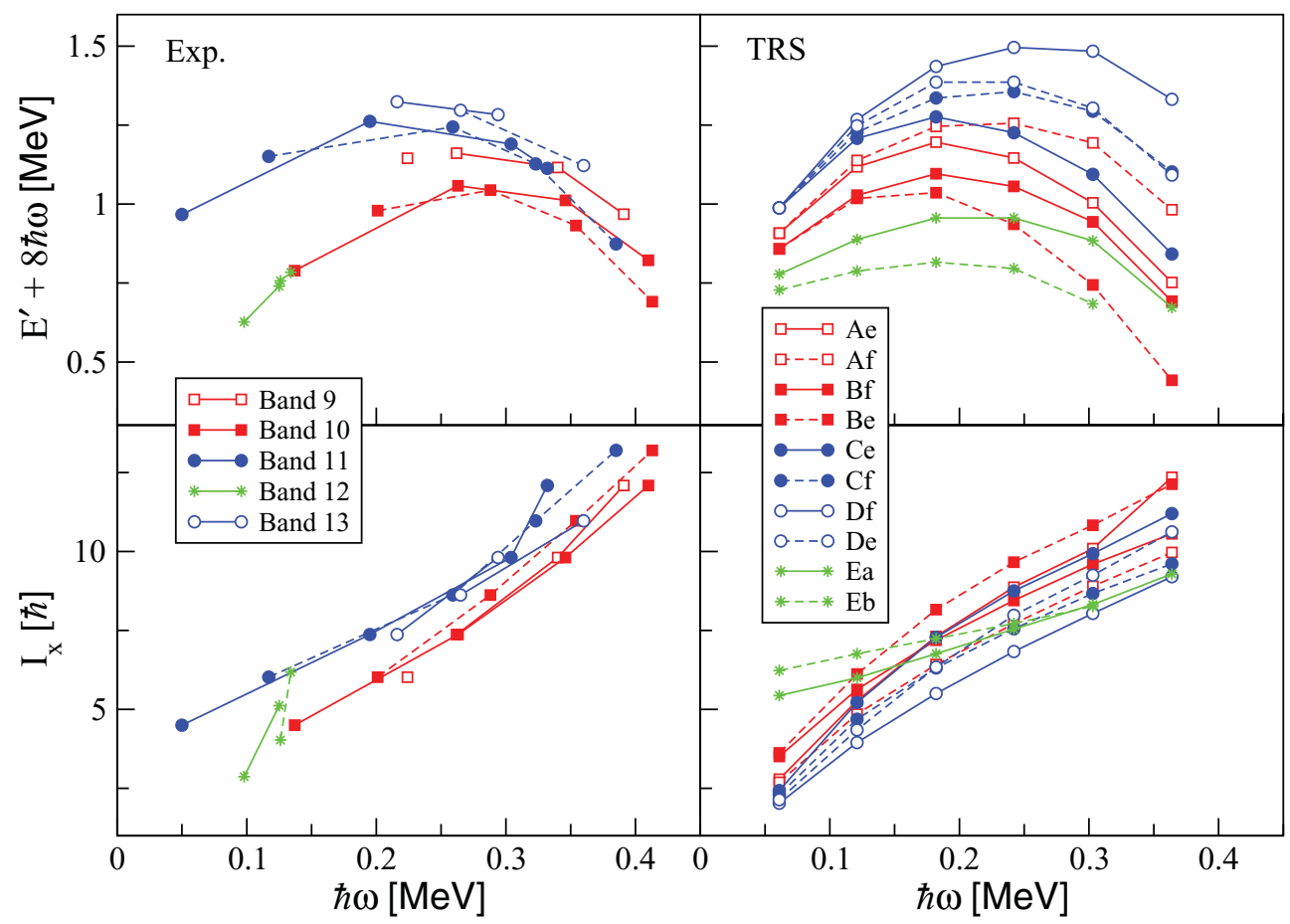

FIG. 8. (Color online) Comparison of the experimental Routhians $\left(E^{\prime}\right)$ and aligned angular momenta $\left(I_{x}\right)$ of the two-quasiparticle negativeparity bands with that predicted by the TRS calculations for the lowest-lying negative-parity configurations.

$\pi[541] 3 / 2^{-}$proton orbital, for which only one $\Delta I=1$ band would be expected.

The four lowest-energy negative-parity TRS configurations without the $h_{11 / 2}$ proton orbital are the $\mathrm{Be}, \mathrm{Bf}, \mathrm{Ae}$, and Af configurations. The relative positions of the predicted Routhians of these configurations are in reasonable agreement with the observed Routhians for bands 9 and 10 . The predicted alignments are also reasonably close to the observed ones. Hence, $(\mathrm{Be}, \mathrm{Bf})$ and $(\mathrm{Ae}, \mathrm{Af})$ are good candidates for the configurations of bands 10 and 9, respectively. These configurations are predicted to have shape parameters around $\left(\beta_{2}=0.2, \gamma=-30^{\circ}\right)$, although with a very shallow minimum as a function of the $\gamma$ parameter, and other local minima $\left(\beta_{2} \approx 0.2, \gamma \approx+20^{\circ}\right)$ and $\left(\beta_{2} \approx 0.2, \gamma \approx-90^{\circ}\right)$ are predicted within a few hundred $\mathrm{keV}$. The A and B TRS states can correspond to either the $\pi[413] 5 / 2$ or the $\pi[411] 3 / 2$ Nilsson orbitals. Because of the several possible shapes, it is difficult to unambiguously identify the corresponding Nilsson orbital; however, the spin value of the bandhead state of band 10 might provide a clue. The $K$ value for the $\pi[411] 3 / 2 \nu[514] 9 / 2$ configuration is expected to be 6 or 3 , with 6 being the favored one, while in the case of the $\pi[413] 5 / 2 \nu[514] 9 / 2$ configuration the expected $K$ values are 7 and 2, with 2 being favored. Thus, bands 9 and 10 probably correspond to the $\pi[411] 3 / 2 v[514] 9 / 2$ Nilsson configuration.

One could expect that the bands 11 and 13 doublet corresponds to the Ce, Cf, Df, and De TRS configurations, which are the next negative-parity configurations not involving the proton $h_{11 / 2}$ orbital. However, the predicted features of these configurations do not agree with the observed features of the bands 11 and 13 doublet. In contrast with the predictions, the observed aligned angular momenta of these bands are not smaller than that observed for bands 9 and 10 , but rather larger by $2 \hbar$. Thus, also in contrast with the predictions, the Routhians of the two doublets converge with increasing rotational frequency instead of diverging. These features are expected for the case of bands in which the $\gamma$ phonon is coupled to the configurations of bands 9 and 10, rather than in the case of the Ce, Cf, Df, and De TRS configurations. This assumption is in line with the fact that the nucleus in the Ae, Af, Bf, and Be TRS configurations is predicted to be rather $\gamma$ soft. Bands in which the $\gamma$ phonon is coupled to the yrast band are observed also in ${ }^{132} \mathrm{Ce}$ [48] and ${ }^{133} \operatorname{Pr}[60]$ with a $\gamma$-phonon energy of around $0.5 \mathrm{MeV}$. In the present case the bandhead energy difference between band 11 and band 10 is close to this value. Therefore, bands 11 and 13 might correspond to the configurations in which a $\gamma$ phonon is coupled to the configurations of bands 9 and 10, or in which these configurations are mixed with the $\mathrm{Ce}, \mathrm{Cf}$, Df, and De TRS configurations.

\section{The negative-parity four-quasiparticle bands}

Bands 5,6 and 7 are $\Delta I=1$ bands linked to each other by several $\gamma$ transitions, suggesting similar configurations. These bands cross bands 9, 10, 11, and 13 at a frequency of around $\hbar \omega=0.3 \mathrm{MeV}$, with an alignment gain of about $8 \hbar$. These crossing frequency and alignment values are close to those predicted by cranked shell-model calculations for the alignment of the first proton pair. On the other hand, the observed $B(M 1) / B(E 2)$ ratios can only be reproduced by the present geometrical model calculations, if the configuration is either $\pi d_{5 / 2}\left(h_{11 / 2}\right)^{2} v h_{11 / 2}$ or $\pi g_{7 / 2}\left(h_{11 / 2}\right)^{2} v h_{11 / 2}$. On the basis of these facts, we can assume that the configurations of bands 
5, 6, and 7 correspond to the above configurations. However, on the basis of the available data we cannot decide which of the above two configurations belong to which band. In the case of band 5, this configuration assignment is in good agreement with that proposed in Ref. [3].

Band 8 is a doubly decoupled negative-parity band. Another doubly decoupled, tentative negative-parity band has already been observed in this nucleus $[29,30]$ and assigned as having $\pi h_{11 / 2} v i_{13 / 2}$ configuration. We also see another band with an intensity of about 1.5 times that of the band 8 intensity; however, we could not link it to the level scheme. The levels belonging to this unlinked band have tentative even spin values in good agreement with the fact that the expected favored signature branch of the $[660] 1 / 2\left(i_{13 / 2}\right)$ neutron orbital is the $\alpha=+1 / 2$ branch, while for the [541]3/2 $\left(h_{11 / 2}\right)$ proton orbital it is the $\alpha=-1 / 2$ branch. The levels of band 8 have odd spin values. Thus this band may correspond to the $\pi h_{11 / 2} v i_{13 / 2}$ configuration either with the $\alpha=+1 / 2$ branch or with the $\alpha=-1 / 2$ branch in both orbitals. The signature splitting is predicted to be smaller for the proton orbital, and thus band 8 probably corresponds to the above configuration with $\alpha=$ $+1 / 2$ signature in both orbitals.

\section{SUMMARY}

The high-spin structure of the ${ }^{134} \mathrm{Pr}$ nucleus has been studied using the ${ }^{116} \mathrm{Cd}\left({ }^{23} \mathrm{Na}, 5 n\right)$ reaction in order to search for new rotational bands and obtain a more complete picture of the structure and special features of this nucleus. The level scheme of ${ }^{134} \mathrm{Pr}$ has been extended from this work. Nine new rotational bands have been found, among which one positive-parity band might be the chiral-partner candidate of the yrare $\pi h_{11 / 2} v h_{11 / 2}$ band, while five of them are the expected two-quasiparticle negative-parity bands. The relative placement of the two previously known isomeric states has been established, and thus the ground state of the nucleus has been unambiguously determined. According to the present results, the $2^{-}$(17-min) state is the ground state and the $6^{-}(11-\mathrm{min})$ state is the excited state at $68 \mathrm{keV}$ above the ground state. The tentatively assigned spin and parity values of the previously known states have been unambiguously determined.

Experimental Routhians and aligned angular momenta, as well as $B(M 1) / B(E 2)$ ratios, have been derived from the data and compared with predictions of total Routhian surface calculations, and results of the geometrical model of Dönau and Frauendorf, respectively. On the basis of these comparisons, and on the basis of comparison with neighboring nuclei, tentative configurations have been assigned to the new bands. According to this assignment, the observed negativeparity two-quasiparticle bands have a $\pi\left(g_{7 / 2} / d_{5 / 2}\right) v h_{11 / 2}$ configuration, and a configuration in which $\pi\left(g_{7 / 2} / d_{5 / 2}\right) \nu h_{11 / 2}$ is coupled to a $\gamma$ phonon. This observation confirms the $\gamma$-soft behavior of ${ }^{134} \mathrm{Pr}$, which is in line with the possible role of shape fluctuations proposed in Ref. [23].

\section{ACKNOWLEDGMENTS}

This work was supported in part by the Hungarian Scientific Research Fund, OTKA (Contract No. K72566), the Natural Sciences and Engineering Research Council of Canada under Contract No. SAPIN/371656-2010, the Bolyai János Foundation, the US National Science Foundation, the US Department of Energy, and the UK Engineering and Physical Sciences Research Council.
[1] S. Frauendorf and J. Meng, Nucl. Phys. A 617, 131 (1997).

[2] V. I. Dimitrov, S. Frauendorf, and F. Dönau, Phys. Rev. Lett. 84, 5732 (2000).

[3] C. M. Petrache, D. Bazzacco, S. Lunardi, C. Rossi Alvarez, G. de Angelis, M. De Poli, D. Bucurescu, C. A. Ur, P. B. Semmes, and R. Wyss, Nucl. Phys. A 597, 106 (1996).

[4] K. Starosta et al., Phys. Rev. Lett. 86, 971 (2001).

[5] A. A. Hecht et al. Phys. Rev. C 63, 051302(R) (2001).

[6] D. J. Hartley et al., Phys. Rev. C 64, 031304(R) (2001).

[7] T. Koike, K. Starosta, C. J. Chiara, D. B. Fossan, and D. R. LaFosse, Phys. Rev. C 63, 061304(R) (2001).

[8] R. A. Bark, A. M. Baxter, A. P. Byrne, G. D. Dracoulis, T. Kibédi, T. R. McGoram, and S. M. Mullins, Nucl. Phys. A 691, 577 (2001).

[9] K. Starosta, C. J. Chiara, D. B. Fossan, T. Koike, T. T. S. Kuo, D. R. LaFosse, S. G. Rohozinski, Ch. Droste, T. Morek, and J. Srebrny, Phys. Rev. C 65, 044328 (2002).

[10] T. Koike, K. Starosta, C. J. Chiara, D. B. Fossan, and D. R. LaFosse, Phys. Rev. C 67, 044319 (2003).

[11] G. Rainovski et al., Phys. Rev. C 68, 024318 (2003).

[12] G. Rainovski et al., J. Phys. G 29, 2763 (2003).

[13] S. Zhu et al., Phys. Rev. Lett. 91, 132501 (2003).

[14] H. C. Jain, S. Lakshmi, and P. K. Joshi, AIP Conf. Proc. 764, 99 (2005).
[15] J. Srebrny, E. Grodner, T. Morek, I. Zalewska, Ch. Droste, J. Mierzejewski, A. A. Pasternak, J. Kownacki, and J. Perkowski, Acta Phys. Pol. B 36, 1063 (2005).

[16] E. Grodner et al., Phys. Rev. Lett. 97, 172501 (2006).

[17] C. Vaman, D. B. Fossan, T. Koike, K. Starosta, I. Y. Lee, and A. O. Macchiavelli, Phys. Rev. Lett. 92, 032501 (2004).

[18] P. Joshi et al., Phys. Lett. B 595, 135 (2004).

[19] P. Joshi et al., Eur. Phys. J. A 24, 23 (2005).

[20] J. A. Alcántara-Núñez et al., Phys. Rev. C 69, 024317 (2004).

[21] J. Timár et al., Phys. Lett. B 598, 178 (2004).

[22] J. Timár, C. Vaman, K. Starosta, D. B. Fossan, T. Koike, D. Sohler, I. Y. Lee, and A. O. Macchiavelli, Phys. Rev. C 73, 011301(R) (2006).

[23] D. Tonev, et al. Phys. Rev. Lett. 96, 052501 (2006).

[24] D. Tonev et al., Phys. Rev. C 76, 044313 (2007).

[25] P. Olbratowski, J. Dobaczewski, and J. Dudek, Phys. Rev. C 73, 054308 (2006).

[26] S. Brant, D. Vretenar, and A. Ventura, Phys. Rev. C 69, 017304 (2004).

[27] C. M. Petrache, G. B. Hagemann, I. Hamamoto, and K. Starosta, Phys. Rev. Lett. 96, 112502 (2006).

[28] C. W. Beausang, L. Hildingsson, E. S. Paul, W. F. Piel Jr., N. Xu, and D. B. Fossan, Phys. Rev. C 36, 1810 (1987).

[29] K. Hauschild et al., Phys. Rev. C 50, 707 (1994). 
[30] M. N. Rao et al., Phys. Rev. C 58, 1367(R) (1998).

[31] Gammasphere Proposal, LBNL-PUB-5202; I. Y. Lee, Nucl. Phys. A 520, 361 (1990).

[32] R. V. F. Janssens and F. S. Stephens, Nucl. Phys. News 6, 9 (1996).

[33] M. Devlin, L. G. Sobotka, D. G. Sarantites, and D. R. LaFosse, Nucl. Instrum. Methods A 383, 506 (1996).

[34] E. S. Paul et al., Phys. Rev. C. (in press).

[35] P. J. Nolan and J. F. Sharpey-Schafer, Rep. Prog. Phys. 42, 1 (1979).

[36] M. Cromaz, T. J. M. Symons, G. J. Lane, I. Y. Lee, and R. W. MacLeod, Nucl. Instrum. Methods A 462, 519 (2001).

[37] D. C. Radford, Nucl. Instrum. Methods A 361, 297 (1995); 361, 306 (1995); [http://radware.phy.ornl.gov].

[38] K. Starosta, D. B. Fossan, T. Koike, C. Vaman, D. C. Radford, and C. J. Chiara, Nucl. Instrum. Methods A 515, 771 (2003).

[39] A. Krämer-Flecken, T. Morek, R. M. Lieder, W. Gast, G. Hebbinghaus, H. M. Jeger, and W. Urban, Nucl. Instrum. Methods A 275, 333 (1989); K. S. Krane, R. M. Steffen, and R. M. Wheeler, Nucl. Data Tables A 11, 351 (1973).

[40] R. Arlt, G. Beyer, V. Fominykh, E. Herrmann, A. Jasinski, H. G. Ortlepp, H. Strusny, H. Tyrroff, and Z. Usmanova, Acta Phys. Pol. B 4, 301 (1973).

[41] Yu.V. Sergeenkov, Nucl. Data Sheets 71, 557 (1994).

[42] A. Gade, I. Wiedenhover, M. Luig, A. Gelberg, H. Meise, N. Pietralla, V. Werner, and P. von Brentano, Nucl. Phys. A 673, 45 (2000).

[43] S. P. Roberts, T. Ahn, K. Starosta, T. Koike, C. J. Chiara, and C. Vaman, Phys. Rev. C 67, 057301 (2003).
[44] T. Kibédi, T. W. Burrows, M. B. Trzhaskovskaya, P. M. Davidson, and C. W. Nestor Jr., Nucl. Instrum. Methods A 589, 202 (2008).

[45] A. A. Sonzogni, Nucl. Data Sheets 103, 1 (2004).

[46] E. S. Paul et al., Nucl. Phys. A 690, 341 (2001).

[47] R. Ma, E. S. Paul, C. W. Beausang, S. Shi, N. Xu, and D. B. Fossan, Phys. Rev. C 36, 2322 (1987).

[48] E. S. Paul et al., Nucl. Phys. A 619, 177 (1997).

[49] F. Dönau and S. Frauendorf, in Proceedings of the Conference on High Angular Momentum Properties of Nuclei, edited by N. R. Johnson (Harwood Academic, New York, 1983), p. 143; F. Dönau, Nucl. Phys. A 471, 469 (1987).

[50] D. C. Radford et al., Nucl. Phys. A 545, 665 (1992).

[51] S. Törmänen et al., Nucl. Phys. A 572, 417 (1994).

[52] A. M. Bizetti-Sona et al., Z. Phys. A 335, 365 (1990); P. Raghavan, At. Data Nucl. Data Tables 42, 189 (1989).

[53] R. Bengtsson and S. Frauendorf, Nucl. Phys. A 327, 139 (1979).

[54] W. Nazarewicz, G. A. Leander, and J. Dudek, Nucl. Phys. A 467, 437 (1987).

[55] W. Nazarewicz, R. Wyss, and A. Johnson, Nucl. Phys. A 503, 285 (1989).

[56] R. Wyss, J. Nyberg, A. Johnson, R. Bengtsson, and W. Nazarewicz, Phys. Lett. B 215, 211 (1988).

[57] L. L. Riedinger et al., Acta Phys. Pol. B 32, 2613 (2001).

[58] G. Andersson et al., Nucl. Phys. A 268, 205 (1976).

[59] C. J. Gallagher Jr. and S. A. Moszkowski, Phys. Rev. 111, 1282 (1958).

[60] L. Hildingsson, C. W. Beausang, D. B. Fossan, and W. F. Piel Jr., Phys. Rev. C 37, 985 (1988). 